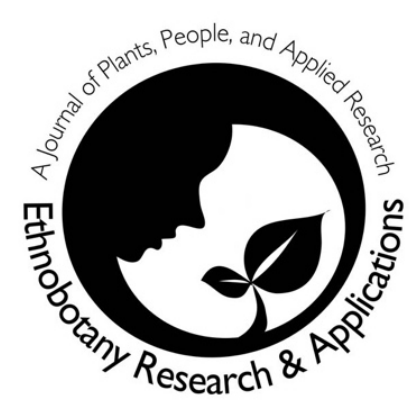

\section{Ethno-medicinal survey of plants used by Magar (Kham) community, Rolpa district, Western Nepal}

\author{
Shanta Budha-Magar, Prabin Bhandari and Suresh \\ Kumar Ghimire
}

\section{Research}

\begin{abstract}
Background: Medicinal plants (MP) are the primary source of health care in developing countries like Nepal. Specially, indigenous people depend on MP species for their basic livelihood. In this study, we aimed to document MP species and assess their indigenous use among Magar (Kham) community in Western Nepal.
\end{abstract}

Methods: A list of MP species was prepared through empirical ethnobotanical study and their indigenous use was assessed using a set of questionnaires. The data collected was generalized for MP species diversity, use categories, and life forms. Informant consensus factor (Fic), Use Frequency (UF) and Use Value (UV) were used to find the cultural importance of MP species.

Results: We recorded $82 \mathrm{MP}$ species, belonging to 45 families and 75 genera of which nine species cited in Magar (Kham) community are ethnobotanically new to Nepal. Informant consensus factor value was found to be high (1) for eight ailment categories; cancer, warts, pneumonia, dandruff, frightening, anesthetic, skin disease, eye irritation and leeches. Based on use value index, the most important ethnomedicinal species were Bergenia ciliata, Swertia chirayta, Acorus calamus and Aconitum gammiei.

Conclusions: This study provides complete document of indigenous uses of MP species among Magar (Kham) community of Thabang Rural Municipality (RM), Rolpa district. Nine MP species were reported with new uses for the first time in Nepal. There is further possibility of recording of new use reports in other villages adjoining to the present studied Magar Kham community. The species with high cultural values must be tested further for their pharmacological properties.
Key words: Rolpa district, Jaljala Mountain, Indigenous Uses, Quantitative ethnobotany, Use Value Index

\section{Correspondence}

Shanta Budha-Magar ${ }^{1 *}$, Prabin Bhandari $^{2}$ and Suresh Kumar Ghimire ${ }^{3}$

${ }^{1}$ City Campus, Auckland University of Technology, Auckland, New Zealand

${ }^{2}$ State Key Laboratory of Systematics and Evolutionary Botany, Institute of Botany, CAS

${ }^{3}$ Central Department of Botany, Tribhuvan

University Kirtipur, Kathmandu, Nepal

*Corresponding Author: shantabmgr@gmail.com

\section{Ethnobotany Research \& Applications}

19:18 (2020)

\section{Background}

The term "ethnobotany" was first given by John Harshberger in 1896 to specify use of plants by aboriginal people. It is the study of relationship between aboriginal society and their plant environment (Schults 1962). It is also defined as the science of people's interaction with plants (Bennett 1996). It deals with the study of relationship of people with plants and the documentation of indigenous knowledge on how local plant resources are utilized by the ethnic groups or communities. The science of ethnobotany includes the understanding of knowledge system by using both the anthropological and botanical approaches (Ford 1978; Davis 1995).

Traditional medicine has been the focus for the primary health care in Nepal and rest of the world since time immemorial. It has been used in either explicably or inexplicably in diagnosis, prevention or elimination of a physical, mental or social disease 
based on past experiences or observation from generation to generation (WHO 2011). MP species are main element in traditional system of healing in developing countries which have been an integral part in history and cultural practices of people. In countries like Nepal, they have been especially valuable in the rural areas where majority (about $80 \%$ ) of people depend for their primary health care (WHO 2011, UNEP 2012, Sathiyaraj et al. 2015). Traditionally, MP species are used as antidote to cure and prevent cuts, wounds, burns, allergies, insect/snake bites and other conditions such as fever, and eye irritation (Shrestha \& Dillion 2003, Mahato \& Chaudhary 2005, Malla \& Chhetri 2009). Furthermore, people use MP species in treatment of illness such as cancer, diabetes, gastrointestinal disorder and skin diseases (Heinrich \& Gibbons 2001, Rokaya et al. 2014, Kunwar et al 2014, Balaraju et al. 2015). Besides, people in rural areas harvest MP species for their economic subsistence too (Olsen 2003).

Moreover, MP species are the novel components and important sources of phytochemicals and antioxidants for drug production in pharmaceutical industries (Cunningham et al. 2008, Malla and Chhetri 2009, De Boer \& Cotingting 2014). About one-third of currently available drugs come from natural products that have a plant origin (Stroh 2000). With an application of developing advance technology in biological activity for screening like high throughput screening and chemical analysis, there have increased interests of researchers towards naturally derived compounds. In this scenario, ethnomedicinal plant survey and recording their subsistence uses will definitely contribute to the identification of MP species (Albuquerque et al. 2013). On other hand, ethnomedicinal study analyzes the results of traditional use of plants together with the cultural context in which the plants and plant parts are used and contribute by its various approaches to preserve, promote and conserve indigenous knowledge (Alexiades 2003).

In documentation of indigenous knowledge and improvement of traditional compilation-style of ethnobotanical studies, there has been increasing incorporation of quantitative research methods in data collection, processing, and interpretation (Hoffman \& Gallaher 2007). One of such key components in quantitative research is relative importance of plant taxa to different human groups. The relative importance such as use-value indices are elaborated based on the agreement or disagreement in the use of plant species. This depends on the geographical focus of survey, vital use of plant by informant number who mention its usefulness (Tardio \& Santayana 2008). Thus, a plant with low importance in broad geographical region could be very important for the people of a small geographical region. Hence, quantitative data thus obtained can be used for macro scale comparison for further study of both biological and cultural diversity (Begossi 1996).

In Nepal, both qualitative and quantitative documentation on use of plants related to ethnomedicine were carried out by several botanists after publication of a paper on medicinal and food plants of eastern Nepal by Banerji in 1955 (Manandhar 2002). Some major studies worked out are; Manandhar 1985, 1986, 1990, 1993, 1995a, 1995b, 2002, Mahato \& Chaudhary 2003, Shrestha \& Pradhan 1986, Shrestha et al. 1998, Ghimire et al. 2000, Lama et al. 2001, Baral \& Kurmi 2006, Shrestha \& Dillion 2003, Mahato \& Chaudhary 2005, Malla \& Chhetri 2009, Uprety et al. 2010, Kunwar et al. 2013 and Singh et al. 2018. However, many communities in different parts of the country are still unexplored. Magar community mostly living in Himalayas or mountains from east to west is one of the least explored community. Only few ethnobotanical studies have been conducted among this community in several parts of Nepal such as; Sapkota 2008, Ale et al. 2009, Acharya 2012, Thapa 2012 and Singh et al. 2018. Among Magar community, Magar (Kham) community of Rolpa district is unmapped community regarding ethnobotanical knowledge, which constitutes $44 \%$ of the district (CBS 2011). They are involved in agriculture and hence highly dependent on local resources specially on MP for their basic health care requirements. But, ethnobotanical study regarding their indigenous knowledge is almost nil. Therefore, we aimed to document indigenous knowledge of this community on use of medicinal plants in Rolpa district, Western Nepal.

\section{Materials and methods}

\section{Study area}

This present study was conducted in ward one and two of Thabang RM in mid-mountain zone of Rolpa district western Nepal (Figure 1). The district is surrounded by Dang to South, Pyuthan to East, Rukum to North and Salyan to West. Geographically, Rolpa lies between $28.8^{0}$ to $28.38^{\circ} \mathrm{N}$ latitudes and $82.10^{\circ}$ to $83.90^{\circ} \mathrm{E}$ longitudes with an elevation range of 700-3600 m asl (meter above sea level). However, present study Thabang RM lies between $28^{0} 30^{\prime} 29.92$ " to $28^{0} 23^{\prime} 52.92^{\prime \prime} \mathrm{N}$ latitude and $82^{\circ} 42^{\prime} 06.32^{\prime \prime}$ to $82^{\circ} 44^{\prime} 43.73^{\prime \prime} \mathrm{E}$ longitude and the elevation ranges from $2000 \mathrm{~m}$ to $3580 \mathrm{~m}$ asl. This area is characterized by the monsoonal type of climate (mean annual precipitation; $1569.7 \mathrm{~mm}$ ). Mean annual maximum temperature (Tmax.) varies from $19.60^{\circ} \mathrm{C}$ to $35.12^{\circ} \mathrm{C}$ and minimum temperature 
(Tmin.) varies from $5.30^{\circ} \mathrm{C}$ to $22.86{ }^{\circ} \mathrm{C}$ (Source: Department of Hydrology and Meteorology, GoN, Kathmandu). This topographic and climatic variability in Jaljala Mountain area have created complex ecological gradients within a small area allowing a great diversity of ecosystems; forests, grasslands or meadows, and wetlands. This zone is characterized by typical assembles of both western and eastern Himalayan elements of flora and fauna. Main inhabitants are Magar (Kham) people of Athâra magarât followed by occupational groups (Bishowkarma and Nepali). Agriculture (animal husbandry and crop production) is the main livelihood strategy adopted in the region. Besides this, collection of Non-timber forest products (NTFPs) including medicinal and aromatic plants is other source of Magar (Kham) people's income.

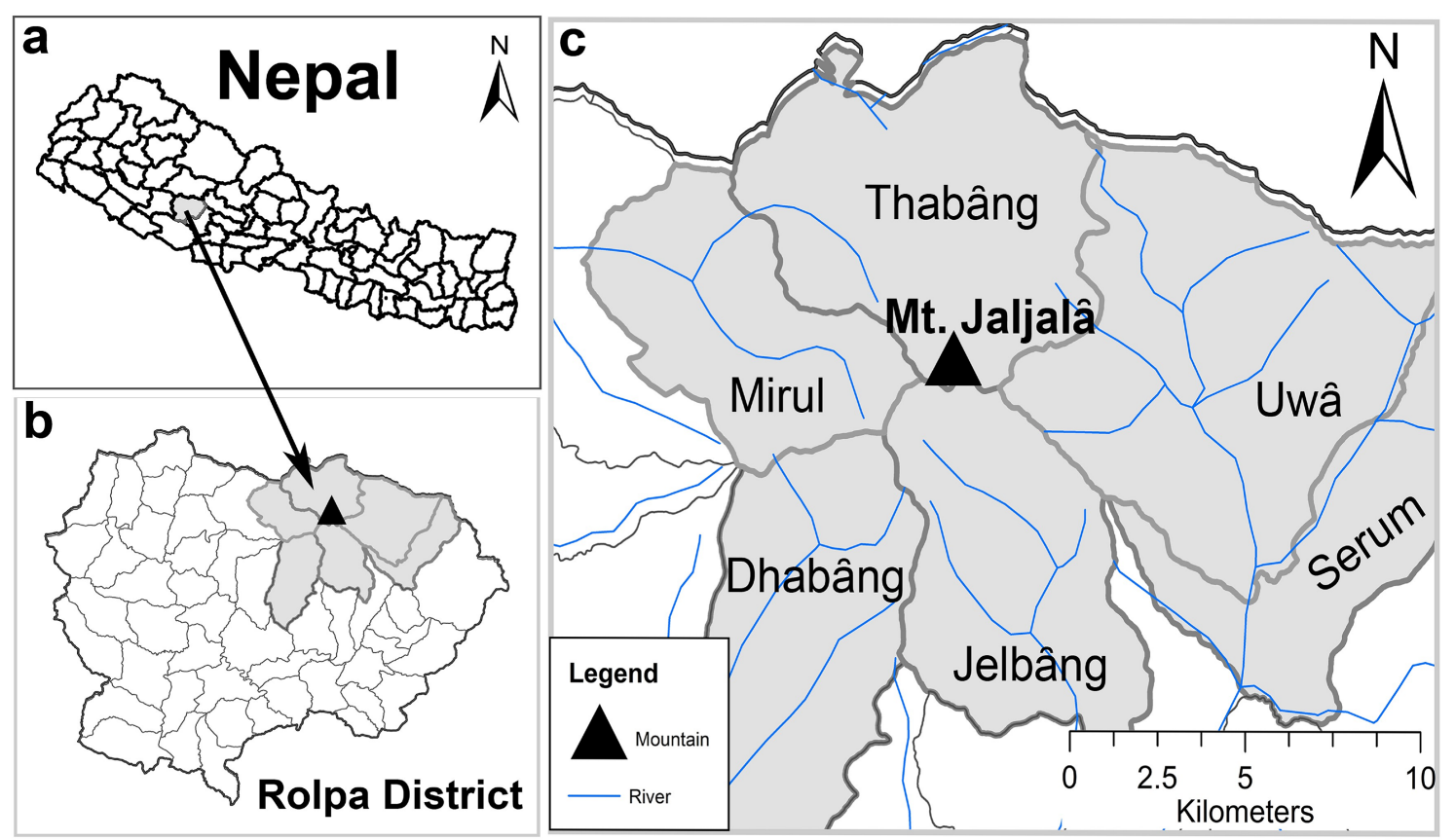

Figure 1. Map of Study area (a) Nepal (b) Rolpa District (c) Jaljala mountain, with surrounding VDCs (Source: Department of Survey, GoN)

Field work and ethnobotanical data collection

An ethnobotanical reconnaissance and data collection were conducted in two field visits in September 2014 and April 2015. Village heads, healers, herders and forest dwellers were invited to participate in FGD (Focus Group Discussion). But general informants were randomly selected, irrespective of age, occupation, sex, religion, education level and castes for interview. Ethical issues were made clear with local people and traditional healers of the communities as established by CBD (Conventional of Biological Diversity) erstwhile the group discussion and interview to share their knowledge. Then, ethnomedicinal data were collected based on an interaction with indigenous people through FGD and direct observation in the field and in-depth data was collected through individual interview

\section{Focus Group Discussion (FGD)}

A total of four Focus Group Discussion (FGD) were conducted in four different villages (Phuntibang, Kherbang, Thabang and Surbang). In each discussion, there were 20-30 people including traditional faith healers, village heads and other local people from different sex and age. The participants were selected based on their rich indigenous knowledge and long-term experience on utilization of plants as well as their living period in the study area as recommended by local administration and district forest officers. During FGD, basic ethnomedicinal information of medicinal plants regarding on their status, uses and priority species were listed. Two resource persons were chosen among participants in FGD conducted in Phuntibang village as recommended by them.

\section{Interviews}

We collected an in-depth information through individual interview employing a set of semistructured questionnaires. We randomly interviewed a total of 50 [(30 male and 20 female) including 5 Jhankries - the traditional healers (4 male and 1 female)]. Our data set of questionnaires covered the respondents' backgrounds namely, the name, age, sex, occupation, level of education, annual income, MP plant taxa, their habitat and distribution, major use categories, part(s) used, mode of collection, 
local status, trade, strategy for mainstay and people's perception on conservation (Additional file 1).

\section{Onsite observation}

Furthermore, transect walk was made to identify local habitat distribution zone of locally used MP species. This comprised of walking along the elevation gradient from 2000 to 3580 meter above sea level in different aspects. During transect walk, plant samples were collected and were crosschecked to confirm the plant use. Two resource persons were involved in the transect walk for collecting locally cited useful plant specimens.

\section{Identification, nomenclature and reliability test of uses}

The collected specimens were pressed, dried, mounted and preserved based on standard methods as given by (Forman \& Bridson 1989). The specimens were identified with the help of standard literatures (Hooker 1872, Hara et al. 1978, 1982, Hara \& Williams 1979, Grierson \& Long 1983; Press et al. 2000, Pearce \& Cribb 2002, Polunin \& Stainton 1984, Polunin et al. 1987, Stainton 1988, Obha et al. 2008, Rajbhandari \& Rai 2017). Furthermore, the species were confirmed by comparing with herbarium specimens deposited at KATH (National Herbarium and Plant Laboratories, Godawori, Lalitpur, Nepal), TUCH (Tribhuvan University Herbarium, Department of Botany, Kirtipur, Kathmandu) and digital specimens deposited at $\mathrm{TI}$ (Herbarium of the University of Tokyo, Japan), K (Herbarium, Royal Botanic Garden, Kew, UK) and E (Herbarium, Royal Botanic Garden, Edinburgh, UK). Then, voucher specimens were deposited in $\mathrm{TUCH}$ and KATH. For nomenclature of species; The plant list (http://www.theplantlist.org/), catalogue of life (www.catalogueoflife.org) and Shrestha et al. 2018, Eflora (www.efloras.org) for Latin name and Nepali name and EPPO Global Database (https://gd.eppo.int/) was followed for English names. Finally, various literatures of recent reviews on flora of Nepal and ethnobotany (e.g. Lama et al. 2001, Rajbhandari 2001, Manandhar 2002, Baral \& Kurmi 2006, Bhattarai \& Ghimire 2006, Dutta 2007, Ghimire \& Pyakurel 2008, Rokaya et al. 2010, Shrestha et al. 2018) were consulted to compare plant uses.

\section{Data analysis}

Both qualitative and quantitative techniques were used to document MP species, compare their uses and cultural importance. Quantitative analyses, especially cultural importance is of great scientific interest as they reflect the cultural value systems, and they aid in the conservation of the biodiversity (Byg \& Baslev 2001) have been used. The data collected through interview was generalized qualitatively for documentation of MP species diversity, life forms and analyzed using three different quantitative tools: Informant consensus factor (Fic), Use Frequency (UF) and Use Values (UV). The Fic was calculated to estimate use variability of plant species and determine which species are particularly interesting (Trotter \& Logan 1986, Heinrich et al. 1998). Fic values range from 0 to 1 . High Fic values are obtained when only one or few plant species are reported to be used by high proportion of informants for particular use, but low Fic values indicate that informants disagree over which plant species to use. Thus, high Fic value can be used to point out interesting plant species for further research. Fic was calculated using the following formula:

$$
\text { Fic }=\frac{\text { Nur }- \text { Nt }}{\text { Nur }-1}
$$

Where, Nur is the number of informants using particular plant species for particular use category and $\mathrm{Nt}$ is the total number of plant species used by all informants for that particular use category.

Similarly, use frequency (UF) was calculated in order to find out the frequency of use of medicinal plant species. It is high when many informants cite the given plant species and approach to zero when only few informants cite a given plant species. It does not show whether a plant species is used for single or multiple purposes. UF was calculated using the following formula used by Tardio \& Santayana 2008:

$$
\mathrm{UF}=\frac{\mathrm{U}}{\mathrm{n}}
$$

Where $U$ is the number of informants who cite the given plant species and $n$ is the total number of informants interviewed in the survey.

Likewise, use value (UV) was calculated in order to find out the importance of medicinal plant species (Arulappan et al. 2015). It is high when a given species is cited for many categories and approach to zero when a species is cited for a single or few categories. It shows whether a plant species is used for single or multiple purposes. UV was calculated using the following formula given by (Philips \& Gentry 1993):

$$
\mathrm{UV}=\frac{\sum \mathrm{Uc}}{\mathrm{n}}
$$

Where Uc is the number of uses mentioned by each informant for a given species and $n$ is the total number of informants.

\section{Results}

Diversity of medicinal plants and their indigenous uses

In present study, a total of 82 MP species out of 175 useful species were recorded, belonging to 45 families and 75 genera based on empirical 
Ethnobotany Research and Applications

Table 1. Medicinal plant species and their indigenous uses in Magar Kham people reported in study area. ( $\dagger=$ Voucher specimen not collected)

\begin{tabular}{|c|c|c|c|c|c|c|c|c|c|c|c|}
\hline Botanical name & $\begin{array}{l}\text { Nepali } \\
\text { name }\end{array}$ & $\begin{array}{l}\text { English } \\
\text { name }\end{array}$ & $\begin{array}{l}\text { Local } \\
\text { name } \\
\text { (Magar) }\end{array}$ & $\begin{array}{l}\text { Life } \\
\text { form }\end{array}$ & $\begin{array}{l}\text { Parts } \\
\text { used }\end{array}$ & $\begin{array}{l}\text { Use } \\
\text { category }\end{array}$ & UV & UF & $\begin{array}{l}\text { Collection } \\
\text { number } I \\
\text { Herbarium }\end{array}$ & $\begin{array}{l}\text { Present findings } \\
\text { (indigenous uses) }\end{array}$ & $\begin{array}{l}\text { Previous } \\
\text { findings }\end{array}$ \\
\hline \multicolumn{12}{|c|}{ FERNS AND FERN ALLIES } \\
\hline $\begin{array}{l}\text { Equisetaceae } \\
\text { Equisetum } \\
\text { arvense* } \mathrm{L} \text {. }\end{array}$ & & $\begin{array}{l}\text { Common } \\
\text { horsetail }\end{array}$ & Mikrop & Herb & RtSh & Md & 0 & 0 & $\begin{array}{l}88 \\
\text { TUCH }\end{array}$ & $\begin{array}{l}\text { Root and stem pieces } \\
\text { are mixed with algae } \\
\text { and Rumex acetosella } \\
\text { roots, and grunt then } \\
\text { wrapped on cotton and } \\
\text { hang around child's neck } \\
\text { during fever. }\end{array}$ & \\
\hline $\begin{array}{l}\text { Pteridaceae } \\
\text { Cheilanthes } \\
\text { albomarginata } \\
\text { C.B. Clarke }\end{array}$ & & & $\begin{array}{l}\text { Dumni } \\
\text { sinkâ }\end{array}$ & Herb & Lf & $\mathrm{Md}$ & 1 & 0.02 & $\begin{array}{l}95 \\
\text { TUCH }\end{array}$ & $\begin{array}{l}\text { Leaf paste is used } \\
\text { externally in cuts and } \\
\text { wounds. }\end{array}$ & $\begin{array}{l}\text { Rh; medicine (2) } \\
\mathrm{Wp}, \mathrm{Rh}, \mathrm{Lf}: \\
\text { medicine }(1,9)\end{array}$ \\
\hline $\begin{array}{l}\text { Selaginellaceae } \\
\text { Selaginella } \\
\text { biformis A. Braun } \\
\text { ex Kuhn }\end{array}$ & & & $\mathrm{Pa}$ & Herb & Lf & Md & 1 & 0.06 & $\begin{array}{l}68 \\
\text { TUCH }\end{array}$ & $\begin{array}{l}\text { Plant paste is applied on } \\
\text { cuts and wounds for } \\
\text { quick healing. }\end{array}$ & $\begin{array}{l}\text { Lf, Rh: medicine } \\
\text { (2) }\end{array}$ \\
\hline \multicolumn{12}{|l|}{ GYMNOSPERMS } \\
\hline $\begin{array}{l}\text { Pinaceae } \\
\text { Pinus wallichiana } \\
\text { A.B. Jacks. }\end{array}$ & $\begin{array}{l}\text { Gobre } \\
\text { sallo }\end{array}$ & $\begin{array}{l}\text { Himalayan } \\
\text { pine }\end{array}$ & Dhupi & Tree & $\begin{array}{l}\text { Wd } \\
\mathrm{Rn}\end{array}$ & $\begin{array}{l}\text { FWTm } \\
\text { Md }\end{array}$ & 1 & 0.22 & $\begin{array}{l}97 \\
\text { TUCH }\end{array}$ & $\begin{array}{l}\text { Woods are used as } \\
\text { fuelwood and timber. } \\
\text { The resin is applied } \\
\text { externally to treat skin } \\
\text { fracture. }\end{array}$ & $\begin{array}{l}\text { Rn: medicine } \\
(1,5), W d, R n: \\
\text { material, } \\
\text { commercial }(2,3) \text {; } \\
\text { Rn: medicine (1), } \\
\text { Wd: religious (8) }\end{array}$ \\
\hline $\begin{array}{l}\text { Taxaceae } \\
\text { Taxus contorta } \\
\text { Griff. }\end{array}$ & Loth sallo & $\begin{array}{l}\text { Western } \\
\text { Himalayan } \\
\text { yew }\end{array}$ & $\begin{array}{l}\text { Jham } \\
\text { chettri }\end{array}$ & Tree & Sh & $\begin{array}{l}\text { FWTm } \\
\text { Fd } \\
\text { Md }\end{array}$ & 1 & 0.12 & $\begin{array}{l}431 \\
\text { KATH/ } \\
\text { TUCH }\end{array}$ & $\begin{array}{l}\text { Wood pieces simply } \\
\text { used as tea or are } \\
\text { boiled, and decoction } \\
\text { obtained is drunk to treat } \\
\text { cancer. Also, woods are } \\
\text { used as fuelwood and } \\
\text { timber. And, leaves are } \\
\text { used as incense. }\end{array}$ & $\begin{array}{l}\text { Wd, Lf: incense, } \\
\text { medicine (2), } \\
\text { religious (8) }\end{array}$ \\
\hline Botanical name & $\begin{array}{l}\text { Nepali } \\
\text { name }\end{array}$ & $\begin{array}{l}\text { English } \\
\text { name }\end{array}$ & $\begin{array}{l}\text { Local } \\
\text { name } \\
\text { (Magar) }\end{array}$ & $\begin{array}{l}\text { Life } \\
\text { form }\end{array}$ & $\begin{array}{l}\text { Parts } \\
\text { used }\end{array}$ & $\begin{array}{l}\text { Use } \\
\text { category }\end{array}$ & UV & UF & $\begin{array}{l}\text { Collection } \\
\text { number } I \\
\text { Herbarium }\end{array}$ & $\begin{array}{l}\text { Present findings } \\
\text { (indigenous uses) }\end{array}$ & $\begin{array}{l}\text { Previous } \\
\text { findings }\end{array}$ \\
\hline
\end{tabular}


Ethnobotany Research and Applications

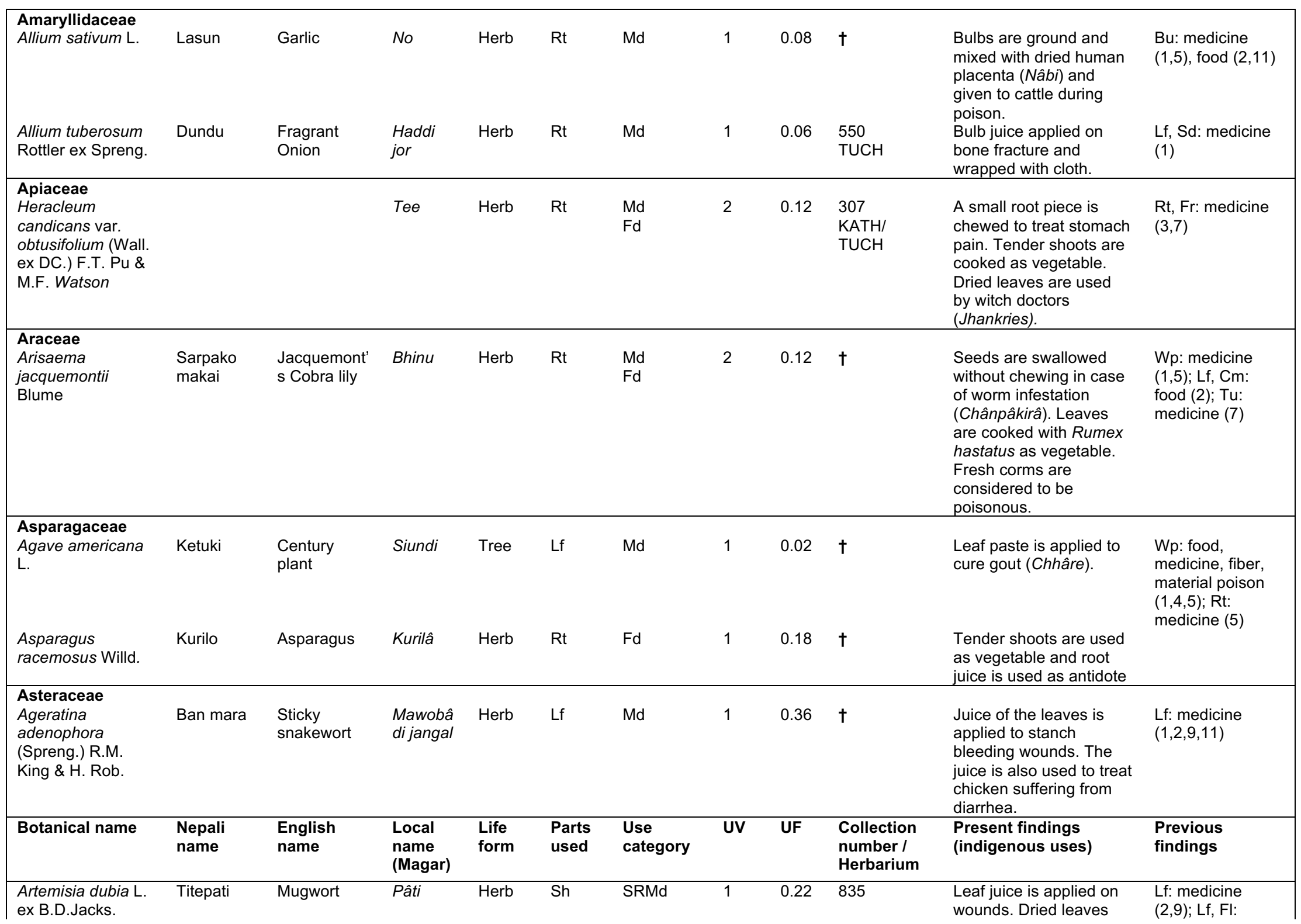


Ethnobotany Research and Applications

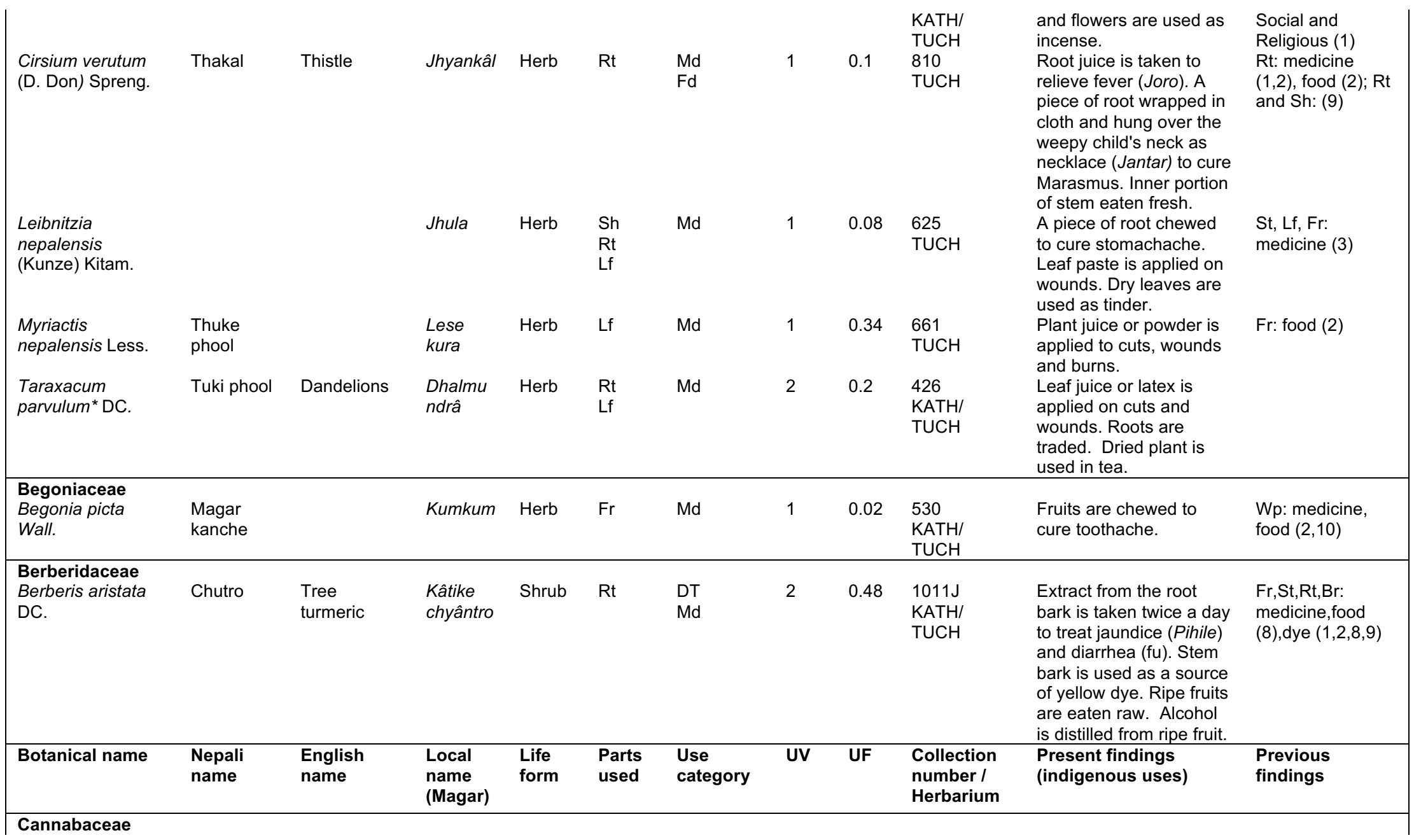


Ethnobotany Research and Applications

\begin{tabular}{|c|c|c|c|c|c|c|c|c|c|c|c|}
\hline Cannabis sativa $\mathrm{L}$. & Bhang & Hemp & Bhângo & Herb & $\mathrm{Br}$ & $\begin{array}{l}\mathrm{Md} \\
\mathrm{Fb} \\
\mathrm{Ol}\end{array}$ & 1 & 0.32 & $\dagger$ & $\begin{array}{l}\text { Bark fiber used for rough } \\
\text { clothes (Bhangaurâ), } \\
\text { sacks, bags. Resinous } \\
\text { exudations of the stem, } \\
\text { and young leaves and } \\
\text { inflorescence yields } \\
\text { intoxicating drug 'Gânâ" } \\
\text { (Attar). Applied on body } \\
\text { to treat pneumonia and } \\
\text { fever. Seeds are roasted } \\
\text { and pickled. Oil } \\
\text { extracted from seeds } \\
\text { used to treat gout. }\end{array}$ & $\begin{array}{l}\mathrm{Br}, \mathrm{Lf}, \mathrm{Sd} \text { : fiber, } \\
\text { medicine, food } \\
(1,2,4,5,9)\end{array}$ \\
\hline $\begin{array}{l}\text { Caprifoliaceae } \\
\text { Morina longifolia } \\
\text { Wall. ex DC. }\end{array}$ & & $\begin{array}{l}\text { Long-leaved } \\
\text { whorl flower }\end{array}$ & $\begin{array}{l}\text { Jhyankâ } \\
\text { tu }\end{array}$ & Herb & Rt & $\mathrm{Md}$ & 1 & 0.02 & $\begin{array}{l}656 \\
\text { KATH/ } \\
\text { TUCH }\end{array}$ & $\begin{array}{l}\text { A small piece of root or } \\
\text { latex, mixed with other } \\
\text { herbs is given to relieve } \\
\text { from fever. }\end{array}$ & $\begin{array}{l}\text { Wp: incense (2); } \\
\text { St, Lf, FI: } \\
\text { medicine (1) }\end{array}$ \\
\hline $\begin{array}{l}\text { Valeriana } \\
\text { hardwickii Wall. }\end{array}$ & $\begin{array}{l}\text { Nakkali } \\
\text { jatamasi }\end{array}$ & & Somâyâ & Herb & $\mathrm{Rh}$ & Md & 1 & 0.1 & $\begin{array}{l}478 \\
\text { KATH/ } \\
\text { TUCH }\end{array}$ & $\begin{array}{l}\text { Root paste is applied on } \\
\text { forehead to treat } \\
\text { headache. Root traded. }\end{array}$ & $\begin{array}{l}\text { Lf, Rh: medicine, } \\
\text { food, religious } \\
\text { (3), Rh: incense, } \\
\text { medicine (8) }\end{array}$ \\
\hline $\begin{array}{l}\text { Caryophyllaceae } \\
\text { Drymaria cordata } \\
\text { (L.) Willd. ex } \\
\text { Schult. }\end{array}$ & Abhiijaalo & Whitesnow & $\begin{array}{l}\text { Nayâ } \\
\text { jungle }\end{array}$ & Herb & Sh, & $\mathrm{Md}$ & 1 & 0.06 & $t$ & $\begin{array}{l}\text { Plant juice is applied to } \\
\text { cure wounds and itches. }\end{array}$ & $\begin{array}{l}\text { Wp, Lf, Ts: } \\
\text { medicine, food } \\
(2)\end{array}$ \\
\hline $\begin{array}{l}\text { Stellaria } \\
\text { himalayensis } \\
\text { Majumdar }\end{array}$ & & & Armâle & Herb & Lf & $\mathrm{Fd}$ & 1 & 0.14 & $\dagger$ & $\begin{array}{l}\text { Leaf juice is applied on } \\
\text { cuts and wounds. } \\
\text { Tender shoots and } \\
\text { leaves cooked as } \\
\text { vegetable. }\end{array}$ & $\begin{array}{l}\text { Wp: medicine } \\
(1,2) ; \text { Ts, Lf: }(2)\end{array}$ \\
\hline $\begin{array}{l}\text { Cornaceae } \\
\text { Cornus capitata* } \\
\text { Wall. }\end{array}$ & Dimur & $\begin{array}{l}\text { Strawberry } \\
\text { dogwood }\end{array}$ & Phuli & Tree & Wd & Fw & 1 & 0.06 & $\begin{array}{l}510 \\
\text { KATH/ } \\
\text { TUCH }\end{array}$ & $\begin{array}{l}\text { Ripe fruits eaten fresh } \\
\text { and believed to cure } \\
\text { headache. They are also } \\
\text { brewed to distill alcohol. } \\
\text { Wood used as fuelwood. }\end{array}$ & \\
\hline $\begin{array}{l}\text { Cucurbitaceae } \\
\text { Solena } \\
\text { heterophylla Lour. }\end{array}$ & Bankankro & & Bidumbâ & $\begin{array}{l}\text { Climb } \\
\text { er }\end{array}$ & Rt & Md & 1 & 0.1 & $\begin{array}{l}450 \\
\text { KATH }\end{array}$ & $\begin{array}{l}\text { Plant juice is given to } \\
\text { treat stomachache. } \\
\text { Fruits are eaten fresh. }\end{array}$ & $\begin{array}{l}\text { Wp, Fr: medicine, } \\
\text { fodder, food ( } 2 \text { ) }\end{array}$ \\
\hline Botanical name & $\begin{array}{l}\text { Nepali } \\
\text { name }\end{array}$ & $\begin{array}{l}\text { English } \\
\text { name }\end{array}$ & $\begin{array}{l}\text { Local } \\
\text { name } \\
\text { (Magar) }\end{array}$ & $\begin{array}{l}\text { Life } \\
\text { form }\end{array}$ & $\begin{array}{l}\text { Parts } \\
\text { used }\end{array}$ & $\begin{array}{l}\text { Use } \\
\text { category }\end{array}$ & UV & UF & $\begin{array}{l}\text { Collection } \\
\text { number } / \\
\text { Herbarium }\end{array}$ & $\begin{array}{l}\text { Present findings } \\
\text { (indigenous uses) }\end{array}$ & $\begin{array}{l}\text { Previous } \\
\text { findings }\end{array}$ \\
\hline $\begin{array}{l}\text { Cyperaceae } \\
\text { Cyperus } \\
\text { cyperoides* }(\mathrm{L} .) \\
\text { Kuntze }\end{array}$ & Karaunte & $\begin{array}{l}\text { Pacific } \\
\text { island } \\
\text { flatsedge }\end{array}$ & $\begin{array}{l}\text { Tolâgâth } \\
\text { â }\end{array}$ & Herb & Rt & Md & 2 & 0.08 & $t$ & $\begin{array}{l}\text { Roots are chewed in } \\
\text { case of gastritis (Gâno). }\end{array}$ & \\
\hline
\end{tabular}


Ethnobotany Research and Applications

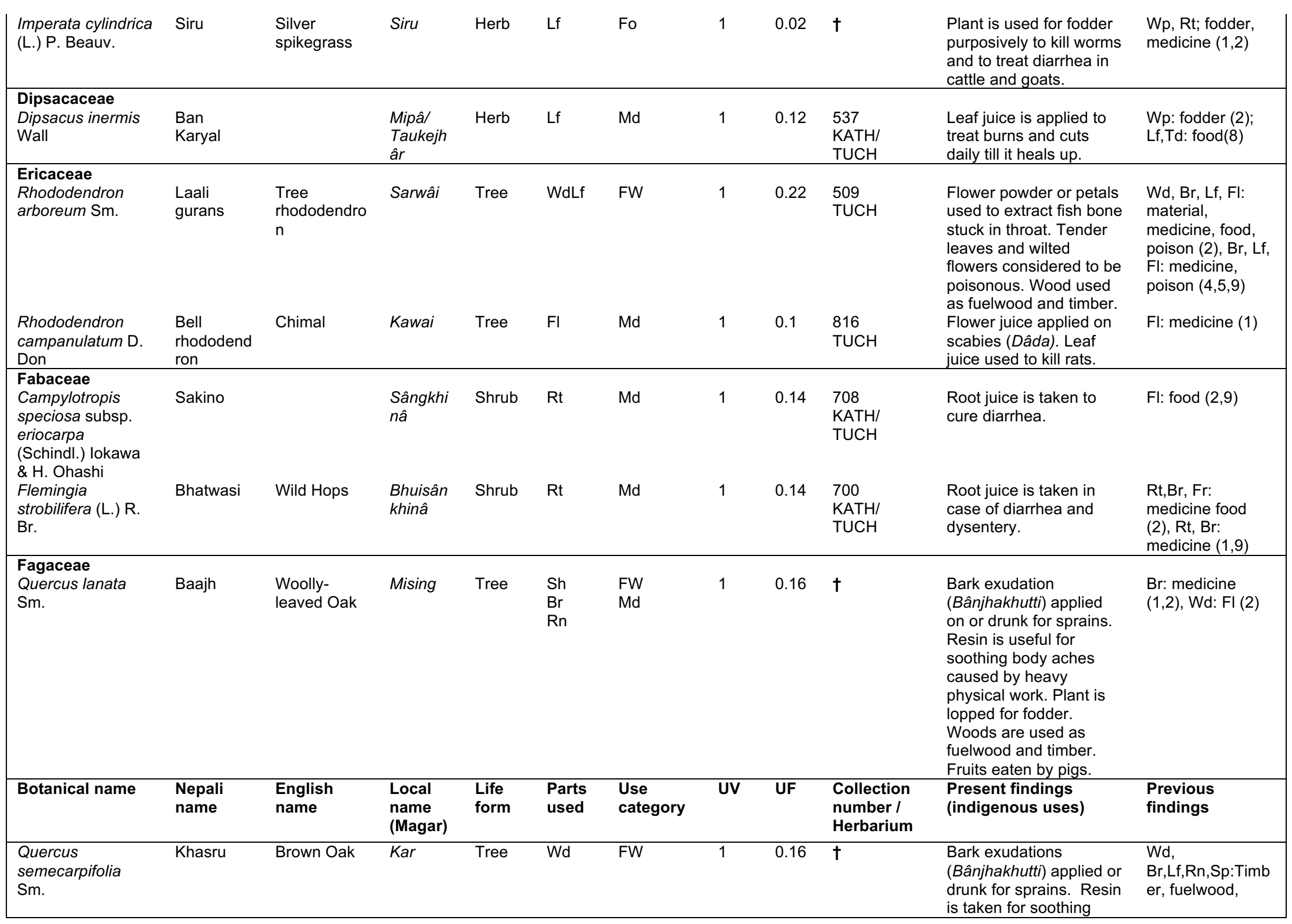


Ethnobotany Research and Applications

\begin{tabular}{|c|c|c|c|c|c|c|c|c|c|c|c|}
\hline & & & & & & & & & & $\begin{array}{l}\text { body aches caused by } \\
\text { heavy physical work. } \\
\text { Plant is lopped for } \\
\text { fodder. Woods used as } \\
\text { fuelwood and timber. }\end{array}$ & $\begin{array}{l}\text { medicine, food } \\
\text { (2) }\end{array}$ \\
\hline $\begin{array}{l}\text { Gentianaceae } \\
\text { Swertia chirayta } \\
\text { (Roxb). Karst. }\end{array}$ & Chiraito & & Runkâ & Herb & $\begin{array}{l}\text { RtShLf } \\
\mathrm{Fl}\end{array}$ & $\mathrm{Md}$ & 2 & 0.48 & $\begin{array}{l}600 \\
\text { TUCH }\end{array}$ & $\begin{array}{l}\text { Plant juice is given to } \\
\text { treat cold, cough, } \\
\text { headache and jaundice. }\end{array}$ & $\begin{array}{l}\text { Wp: medicine } \\
(1,2,3,4,5,9)\end{array}$ \\
\hline $\begin{array}{l}\text { Geraniaceae } \\
\text { Geranium } \\
\text { procurrens }{ }^{*} \text { Yeo }\end{array}$ & & & Châpâ & Herb & Sh, & Md & 1 & 0.04 & $\begin{array}{l}554 \\
\text { KATH/ } \\
\text { TUCH }\end{array}$ & $\begin{array}{l}\text { Plant mixed with other } \\
\text { plants (Giardinia, } \\
\text { Rheum austral, Rumex } \\
\text { and Prunus cerasoides) } \\
\text { pounded and applied on } \\
\text { sprains (Piriu) }\end{array}$ & \\
\hline $\begin{array}{l}\text { Juglandaceae } \\
\text { Juglans regia L. }\end{array}$ & $\begin{array}{l}\text { Sano } \\
\text { Okhar }\end{array}$ & Walnut & Khâsai & Tree & SR & & 1 & 0.24 & $\begin{array}{l}757 \\
\text { TUCH }\end{array}$ & $\begin{array}{l}\text { Bark is chewed in case } \\
\text { of toothache. Also yields } \\
\text { dye and sometime used } \\
\text { as fish poison. Woods } \\
\text { serve as essential item } \\
\text { during funeral. Kernel } \\
\text { eaten raw. }\end{array}$ & Br: medicine (1) \\
\hline $\begin{array}{l}\text { Lamiaceae } \\
\text { Leucosceptrum } \\
\text { canum Sm. }\end{array}$ & Bhusure & & $\begin{array}{l}\text { Phusary } \\
\hat{a}\end{array}$ & Shrub & $\mathrm{Rt}$ & Md & 1 & 0.02 & $\begin{array}{l}806 \\
\text { TUCH }\end{array}$ & $\begin{array}{l}\text { Root paste is applied to } \\
\text { wounds. }\end{array}$ & $\begin{array}{l}\text { Lf, Fr; fodder, } \\
\text { food (2) }\end{array}$ \\
\hline $\begin{array}{l}\text { Prunella vulgaris } \\
\text { L. }\end{array}$ & & $\begin{array}{l}\text { Common } \\
\text { selfheal }\end{array}$ & Dhâkar & Herb & Lf & $\mathrm{Fd}$ & 1 & 0.08 & $t$ & $\begin{array}{l}\text { Plant paste is used to } \\
\text { treat backaches. }\end{array}$ & Wp: medicine (2) \\
\hline $\begin{array}{l}\text { Lauraceae } \\
\text { Lindera neesiana } \\
\text { (Wall. ex Nees) } \\
\text { Kurz }\end{array}$ & Sil timur & & Tiipâ & Tree & $\mathrm{Fr}$ & $\mathrm{Fd}$ & 2 & 0.14 & $\begin{array}{l}697 \\
\text { KATH/ } \\
\text { TUCH }\end{array}$ & $\begin{array}{l}\text { When cattle, eat } \\
\text { poisonous plants, dry } \\
\text { fruits given and also to } \\
\text { remove placenta during } \\
\text { delivery. People eat } \\
\text { fruits in case of stomach } \\
\text { problems and flatulence } \\
\text { and sometime use as } \\
\text { spices in vegetable too. }\end{array}$ & $\begin{array}{l}\text { Rt, Br, Lf, Fr, Sd: } \\
\text { medicine (1,5); } \\
\text { Fr, Sd: medicine } \\
\text { (2,9) }\end{array}$ \\
\hline Botanical name & $\begin{array}{l}\text { Nepali } \\
\text { name }\end{array}$ & $\begin{array}{l}\text { English } \\
\text { name }\end{array}$ & $\begin{array}{l}\text { Local } \\
\text { name } \\
\text { (Magar) }\end{array}$ & $\begin{array}{l}\text { Life } \\
\text { form }\end{array}$ & $\begin{array}{l}\text { Parts } \\
\text { used }\end{array}$ & $\begin{array}{l}\text { Use } \\
\text { category }\end{array}$ & UV & UF & $\begin{array}{l}\text { Collection } \\
\text { number } I \\
\text { Herbarium }\end{array}$ & $\begin{array}{l}\text { Present findings } \\
\text { (indigenous uses) }\end{array}$ & $\begin{array}{l}\text { Previous } \\
\text { findings }\end{array}$ \\
\hline $\begin{array}{l}\text { Melianthaceae } \\
\text { Paris polyphylla } \\
\text { Sm. }\end{array}$ & Satuwa & Love apple & Satawâ & Herb & $\mathrm{Rh}$ & Md & 1 & 0.32 & $836 \mathrm{TUCH}$ & $\begin{array}{l}\text { Rhizome powder in low } \\
\text { amount is applied to } \\
\text { cuts and wounds for } \\
\text { rapid healing. High use } \\
\text { of powder gets plugged }\end{array}$ & $\begin{array}{l}\text { Rh: medicine } \\
(1,2,3,4,8,9), \\
\text { commercial (3) }\end{array}$ \\
\hline
\end{tabular}


Ethnobotany Research and Applications

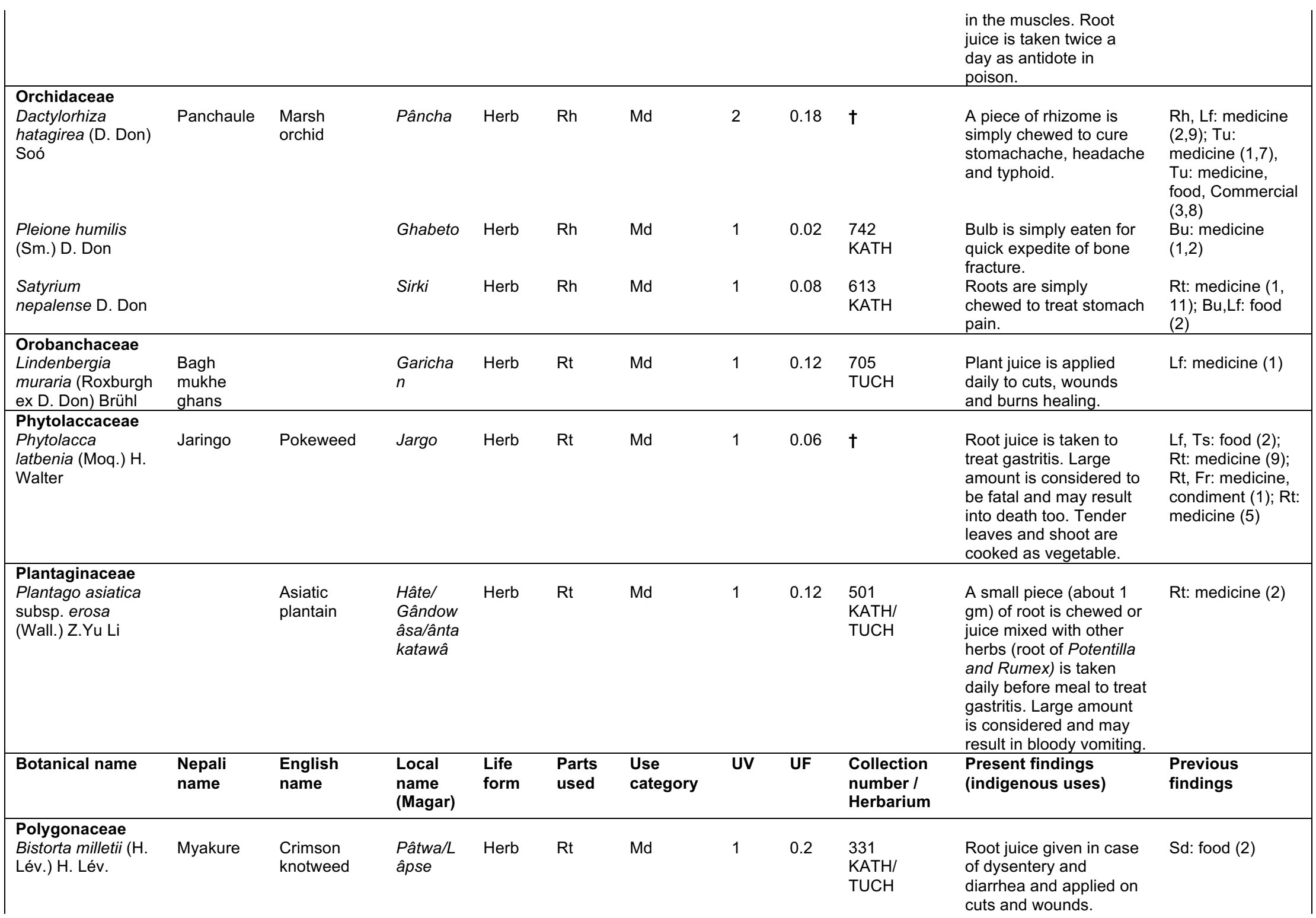


Ethnobotany Research and Applications

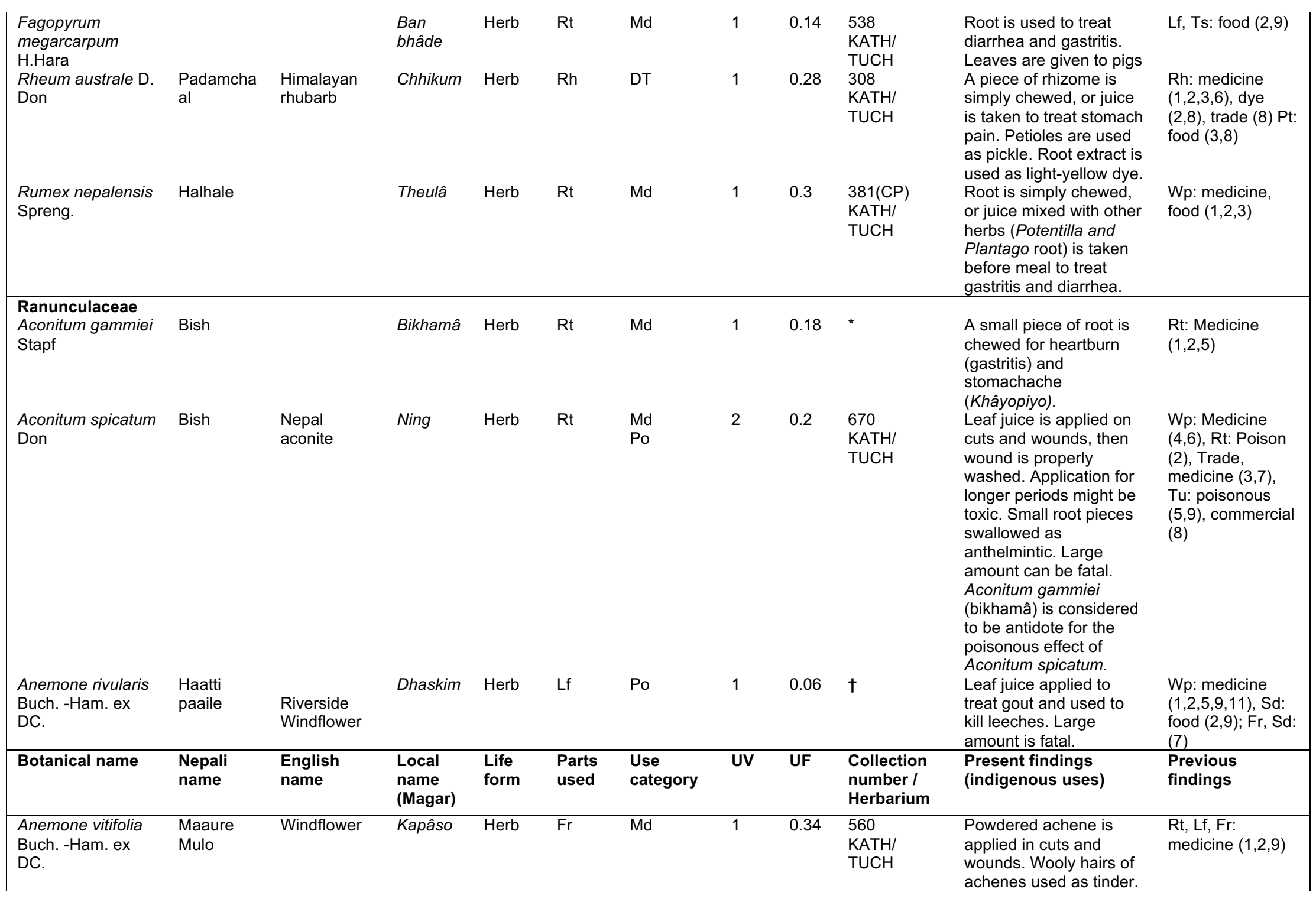


Ethnobotany Research and Applications

\begin{tabular}{|c|c|c|c|c|c|c|c|c|c|c|c|}
\hline $\begin{array}{l}\text { Clematis terniflora* } \\
\text { DC. }\end{array}$ & & $\begin{array}{l}\text { Sweet } \\
\text { autumn } \\
\text { clematis }\end{array}$ & Abijâle & $\begin{array}{l}\text { Climb } \\
\text { er }\end{array}$ & Sh & $\mathrm{Md}$ & 1 & 0.08 & $\begin{array}{l}829 \\
\dagger\end{array}$ & $\begin{array}{l}\text { Aerial part paste is } \\
\text { applied to treat gout. } \\
\text { Whole plant juice is } \\
\text { applied on cloth and is } \\
\text { applied to cure itching } \\
\text { and joint ache. Large } \\
\text { amount is considered to } \\
\text { be fatal. }\end{array}$ & \\
\hline $\begin{array}{l}\text { Delphinium } \\
\text { himalayae Munz }\end{array}$ & & $\begin{array}{l}\text { Himalayan } \\
\text { larkspur }\end{array}$ & Nirmasii & Herb & $\mathrm{Rt}$ & $\mathrm{Md}$ & 1 & 0.1 & $t$ & $\begin{array}{l}\text { A small piece of root is } \\
\text { simply chewed before } \\
\text { breakfast daily for } 2 \text { to } 4 \\
\text { days in case of } \\
\text { headache. Large } \\
\text { amount is fatal. }\end{array}$ & $\begin{array}{l}\text { Rt: Medicine (1), } \\
\text { Tu: medicine } \\
(1,3,8,11) \text {, poison } \\
(1) \text {, Commercial } \\
(3,8)\end{array}$ \\
\hline $\begin{array}{l}\text { Delphinium } \\
\text { grandiflorum }{ }^{*} \mathrm{~L} .\end{array}$ & & $\begin{array}{l}\text { Bouquet } \\
\text { larkspur }\end{array}$ & Atis & Herb & Rt & Md & 1 & 0.02 & $\begin{array}{l}830 \\
\text { KATH/ } \\
\text { TUCH }\end{array}$ & $\begin{array}{l}\text { A small piece of root is } \\
\text { chewed to treat stomach } \\
\text { pain and typhoid each } \\
\text { morning before } \\
\text { breakfast. Root paste is } \\
\text { applied for joint pain. }\end{array}$ & \\
\hline $\begin{array}{l}\text { Delphinium } \\
\text { vestitum Wall. ex } \\
\text { Royle }\end{array}$ & $\begin{array}{l}\text { Mawar- } \\
\text { mul }\end{array}$ & & $\begin{array}{l}\text { Mangrâ } \\
\text { mhul }\end{array}$ & Herb & Rt,Lf & $\mathrm{Md}$ & 2 & 0.28 & $\begin{array}{l}612 \\
\text { KATH/ } \\
\text { TUCH }\end{array}$ & $\begin{array}{l}\text { The root is used as } \\
\text { anthelmintic. It is also } \\
\text { used to poison dogs. } \\
\text { Root and leaf paste } \\
\text { mixed with Aconitum sp. } \\
\text { is applied on wounds } \\
\text { and juice is given to } \\
\text { animal during delivery. }\end{array}$ & $\begin{array}{l}\text { Rt: medicine } \\
(1,2,9,11) \text {, poison } \\
(2)\end{array}$ \\
\hline $\begin{array}{l}\text { Rosaceae } \\
\text { Cotoneaster } \\
\text { microphyllus Wall. } \\
\text { ex Lindl. }\end{array}$ & Khoreto & $\begin{array}{l}\text { Rock-spray } \\
\text { cotoneaster }\end{array}$ & $\begin{array}{l}\text { Sapithâl } \\
\text { â }\end{array}$ & Shrub & $\mathrm{Fr}$ & Fw & 1 & 0.02 & $\begin{array}{l}525 \\
\text { KATH/ } \\
\text { TUCH }\end{array}$ & $\begin{array}{l}\text { Raw fruits are eaten and } \\
\text { believed to cure asthma }\end{array}$ & $\begin{array}{l}\text { Wd, Fr: Fuel, } \\
\text { food (2) }\end{array}$ \\
\hline $\begin{array}{l}\text { Potentilla lineata } \\
\text { Trevir. }\end{array}$ & Bajradanti & & $\begin{array}{l}\text { Ban } \\
\text { mulâ }\end{array}$ & Herb & Rt & $\mathrm{Md}$ & 1 & 0.32 & $\dagger$ & $\begin{array}{l}\text { A piece of root (about } 1 \\
\text { gm) is simply chewed or } \\
\text { juice mixed with root } \\
\text { juice of Potentilla and } \\
\text { Rumex in equal amount } \\
\text { is taken daily before } \\
\text { meal to treat gastritis. } \\
\text { Large amount is } \\
\text { considered to be fatal. }\end{array}$ & Rt: medicine $(1,9)$ \\
\hline Botanical name & $\begin{array}{l}\text { Nepali } \\
\text { name }\end{array}$ & $\begin{array}{l}\text { English } \\
\text { name }\end{array}$ & $\begin{array}{l}\text { Local } \\
\text { name } \\
\text { (Magar) }\end{array}$ & $\begin{array}{l}\text { Life } \\
\text { form }\end{array}$ & $\begin{array}{l}\text { Parts } \\
\text { used }\end{array}$ & $\begin{array}{l}\text { Use } \\
\text { category }\end{array}$ & UV & UF & $\begin{array}{l}\text { Collection } \\
\text { number } / \\
\text { Herbarium }\end{array}$ & $\begin{array}{l}\text { Present findings } \\
\text { (indigenous uses) }\end{array}$ & $\begin{array}{l}\text { Previous } \\
\text { findings }\end{array}$ \\
\hline $\begin{array}{l}\text { Prinsepia utilis } \\
\text { Royle }\end{array}$ & Dhatelo & $\begin{array}{l}\text { Cherry } \\
\text { prinsepia }\end{array}$ & Kaikirâm & Shrub & Sh, & FWMd & 1 & 0.12 & $t$ & $\begin{array}{l}\text { Seed oil is used in case } \\
\text { of joint ache or gout. }\end{array}$ & $\begin{array}{l}\text { Sd: medicine } \\
(1,10)\end{array}$ \\
\hline
\end{tabular}


Ethnobotany Research and Applications

\begin{tabular}{|c|c|c|c|c|c|c|c|c|c|c|c|}
\hline $\begin{array}{l}\text { Prunus cerasoides } \\
\text { Buch. -Ham. ex D. } \\
\text { Don }\end{array}$ & Painyu & $\begin{array}{l}\text { Wild } \\
\text { Himalayan } \\
\text { cherry }\end{array}$ & Paiyâ & Tree & St & SRDTMd & 0 & 0.38 & $\begin{array}{l}\dagger \\
\text { t }\end{array}$ & $\begin{array}{l}\text { Stems are used in } \\
\text { funerals and by Jhânkri. } \\
\text { Barks are cooked and } \\
\text { juice thus extracted is } \\
\text { taken daily in case of } \\
\text { sprains and backaches } \\
\text { (Pireko), swellings and } \\
\text { also used as dye. }\end{array}$ & $\begin{array}{l}\mathrm{Wp}, \mathrm{Br}, \mathrm{Lf}, \mathrm{Sd} \text { : } \\
\text { material, fodder, } \\
\text { medicine (2), Br, } \\
\mathrm{Lf}, \mathrm{Sd} \text { : medicine } \\
\text { (5), Br: medicine } \\
\text { (9); }\end{array}$ \\
\hline $\begin{array}{l}\text { Rosa macrophylla } \\
\text { Lindl. }\end{array}$ & $\begin{array}{l}\text { Bhainsi } \\
\text { kaandaa }\end{array}$ & $\begin{array}{l}\text { Himalayan } \\
\text { Rose }\end{array}$ & $\begin{array}{l}\text { Bhârmâ } \\
\text { se }\end{array}$ & Shrub & Rt & $\mathrm{Md}$ & 1 & 0.04 & $\begin{array}{l}418 \\
\text { KATH/ } \\
\text { TUCH }\end{array}$ & $\begin{array}{l}\text { A small piece of root is } \\
\text { chewed to treat } \\
\text { heartburn and stomach } \\
\text { pain. Large amount is } \\
\text { considered to be fatal. }\end{array}$ & $\begin{array}{l}\text { FI, Lf: medicine, } \\
\text { food, fodder }(2,3) ; \\
\text { Fr: medicine }(7)\end{array}$ \\
\hline $\begin{array}{l}\text { Rubus } \\
\text { hoffmeisterianus* } \\
\text { Kunth \& Bouché }\end{array}$ & & $\begin{array}{l}\text { Hoffmeister } \\
\text { Raspberry }\end{array}$ & Zoosai & Shrub & $\mathrm{Fr}$ & Fd, & 1 & 0.3 & $\begin{array}{l}831 \\
\mathrm{TUCH}\end{array}$ & $\begin{array}{l}\text { Fermented leaf juice } \\
\text { mixed with Pteris sp. is } \\
\text { given to treat Marasmus } \\
\text { and in-appetite. Ripe } \\
\text { fruits are eaten fresh. }\end{array}$ & \\
\hline $\begin{array}{l}\text { Rubiaceae } \\
\text { Galium } \\
\text { asperuloides }\end{array}$ & & & Khasare & Herb & Lf & Md & 1 & 0.1 & $\begin{array}{l}303 \\
\text { KATH/ }\end{array}$ & $\begin{array}{l}\text { Plant juice is used on } \\
\text { cuts and wounds. }\end{array}$ & Wp: medicine (1) \\
\hline $\begin{array}{l}\text { Edgew. } \\
\text { Rubia manjith } \\
\text { Roxb. ex Fleming }\end{array}$ & Majitho & & Khasare & Herb & Lf & Md & 1 & 0.08 & $\begin{array}{l}\text { TUCH } \\
539 \\
\text { TUCH }\end{array}$ & $\begin{array}{l}\text { Leaf juice is applied on } \\
\text { cuts and wounds. }\end{array}$ & $\begin{array}{l}\text { Rt, St: medicine, } \\
\text { dye (2); Lf: } \\
\text { medicine (1), Rt: } \\
\text { dye ( } 8 \text { ) }\end{array}$ \\
\hline $\begin{array}{l}\text { Rutaceae } \\
\text { Zanthoxylum } \\
\text { armatum DC }\end{array}$ & Timur & & Tinbur & Shrub & $\mathrm{Fr}$ & $\mathrm{Fd}$ & 1 & 0.2 & $\dagger$ & $\begin{array}{l}\text { Seeds are chewed in } \\
\text { case of cold, stomach } \\
\text { disorder and poison. } \\
\text { Fruits used as pickle. }\end{array}$ & $\begin{array}{l}\mathrm{Rt}, \mathrm{Br}, \mathrm{Lf}, \mathrm{Fr}, \mathrm{Sd}: \\
\text { medicine, food } \\
(1,2,4,5,9)\end{array}$ \\
\hline $\begin{array}{l}\text { Santalaceae } \\
\text { Viscum album L. }\end{array}$ & Hadchur & $\begin{array}{l}\text { White- } \\
\text { berried } \\
\text { mistletoe }\end{array}$ & Jokhâre & Shrub & $W p$ & $\mathrm{Md}$ & 1 & 0.12 & $\begin{array}{l}779 \\
\text { KATH/ } \\
\text { TUCH }\end{array}$ & $\begin{array}{l}\text { Plant paste is applied on } \\
\text { dislocated and disjointed } \\
\text { bones in sheep / goats. }\end{array}$ & Wp: medicine (1) \\
\hline $\begin{array}{l}\text { Sapindaceae } \\
\text { Aesculus indica } \\
\text { (Wall. ex } \\
\text { Cambess.) Hook. }\end{array}$ & $\begin{array}{l}\text { Ghode } \\
\text { paangro }\end{array}$ & $\begin{array}{l}\text { Horse } \\
\text { Chestnut }\end{array}$ & Pângar & Tree & Sh & FWTmMd & 3 & 0.08 & $\begin{array}{l}782 \\
\text { TUCH }\end{array}$ & $\begin{array}{l}\text { Dry fruit is rubbed } \\
\text { against stone, thus } \\
\text { produced powder is } \\
\text { made paste and applied } \\
\text { to cure Mumps (Bâgale). } \\
\text { Wood used as fuelwood. }\end{array}$ & $\begin{array}{l}\mathrm{Br} \text {, Lf: Medicine, } \\
\text { fodder (1); Sd: } \\
\text { food }(1,2,4,9) \\
\text { medicine (5), Sh, } \\
\mathrm{Lf} \text { food (4), Wd; } \\
\text { Material (4) }\end{array}$ \\
\hline Botanical name & $\begin{array}{l}\text { Nepali } \\
\text { name }\end{array}$ & $\begin{array}{l}\text { English } \\
\text { name }\end{array}$ & $\begin{array}{l}\text { Local } \\
\text { name } \\
\text { (Magar) }\end{array}$ & $\begin{array}{l}\text { Life } \\
\text { form }\end{array}$ & $\begin{array}{l}\text { Parts } \\
\text { used }\end{array}$ & $\begin{array}{l}\text { Use } \\
\text { category }\end{array}$ & UV & UF & $\begin{array}{l}\text { Collection } \\
\text { number } I \\
\text { Herbarium }\end{array}$ & $\begin{array}{l}\text { Present findings } \\
\text { (indigenous uses) }\end{array}$ & $\begin{array}{l}\text { Previous } \\
\text { findings }\end{array}$ \\
\hline $\begin{array}{l}\text { Saxifragaceae } \\
\text { Bergenia ciliata } \\
\text { Sternb. }\end{array}$ & $\begin{array}{l}\text { Paakhanb } \\
\text { ed }\end{array}$ & Bergenia & $\begin{array}{l}\text { Hângaw } \\
\text { o }\end{array}$ & Herb & $\mathrm{Rh}$ & Md & 3 & 0.42 & 775 & $\begin{array}{l}\text { Rhizome juice is taken } \\
\text { to treat, typhoid }\end{array}$ & $\begin{array}{l}\text { Wp, Fl,Rh,Lf: } \\
\text { medicine }\end{array}$ \\
\hline
\end{tabular}


Ethnobotany Research and Applications

\begin{tabular}{|c|c|c|c|c|c|c|c|c|c|c|c|}
\hline & & & & & & & & & $\begin{array}{l}\text { KATH/ } \\
\text { TUCH }\end{array}$ & $\begin{array}{l}\text { (Ghâmjoro), diarrhea, } \\
\text { dysentery and vomiting. } \\
\text { A small piece of rhizome } \\
\text { is chewed in case of } \\
\text { stomach pain, headache } \\
\text { and menstruation } \\
\text { disorder. Large piece is } \\
\text { fatal. }\end{array}$ & $\begin{array}{l}(2,4,5,7,9) ; \mathrm{Rh}, \mathrm{Sd} \text {, } \\
\text { Wp: medicine } \\
\text { (1,8); commercial } \\
\text { (3)Rh, Rt:medicin } \\
\text { e (4,11); Rt: } \\
\text { medicine (5) }\end{array}$ \\
\hline $\begin{array}{l}\text { Scrophulariaceae } \\
\text { Verbascum } \\
\text { thapsus L. }\end{array}$ & $\begin{array}{l}\text { Bandarpuc } \\
\text { hhre }\end{array}$ & Candlewick & Yume & Herb & $\mathrm{Rt}$ & $\mathrm{Md}$ & 1 & 0.04 & $\begin{array}{l}527 \\
\text { TUCH }\end{array}$ & $\begin{array}{l}\text { Root juice is given to } \\
\text { child to treat diarrhea, } \\
\text { dyspepsia and fever. }\end{array}$ & $\begin{array}{l}\text { Wp,Rt: medicine, } \\
\text { poison }(1,2,9) ; \text { Lf, } \\
\text { Rt, St,FL; } \\
\text { medicine, poison } \\
\text { (5); Lf, St,FI: } \\
\text { medicine (7) }\end{array}$ \\
\hline $\begin{array}{l}\text { Simaroubaceae } \\
\text { Brucea javanica } \\
\text { (L.) Merr. }\end{array}$ & Bhakimlo & Kosam & $\begin{array}{l}\text { Bhakimb } \\
\text { lâ }\end{array}$ & Tree & $\mathrm{Fr}$ & $\mathrm{Fd}$ & 1 & 0.14 & $\begin{array}{l}566 \\
\text { KATH/ } \\
\text { TUCH }\end{array}$ & $\begin{array}{l}\text { Fruit decoction is taken } \\
\text { to cure diarrhea and } \\
\text { dysentery. Ripe fruits } \\
\text { are eaten either raw or } \\
\text { pickled }\end{array}$ & $\begin{array}{l}\text { Fr: medicine }(1, \\
10)\end{array}$ \\
\hline $\begin{array}{l}\text { Thymelaeaceae } \\
\text { Daphne bholua } \\
\text { Buch.-Ham. ex D. } \\
\text { Don }\end{array}$ & Lokata & $\begin{array}{l}\text { Jacqueline } \\
\text { Postill }\end{array}$ & $\begin{array}{l}\text { Barruwâ } \\
\text { / } \\
\text { Ratuwâc } \\
\text { hâro }\end{array}$ & Shrub & Rt & $\begin{array}{l}\mathrm{Md} \\
\mathrm{Fb}\end{array}$ & 0 & 0.16 & $\begin{array}{l}788 \\
\text { KATH/ } \\
\text { TUCH }\end{array}$ & $\begin{array}{l}\text { Root juice mixed with } \\
\text { other herbs is taken to } \\
\text { treat fever. Bark is } \\
\text { extracted for fiber. }\end{array}$ & $\begin{array}{l}\mathrm{Rt}, \mathrm{Br}, \mathrm{Lf}: \\
\text { medicine, } \\
\text { material, poison } \\
(2,4) ; \mathrm{Rt}: \\
\text { medicine }(9) ; \mathrm{Rt}, \\
\mathrm{Br}:(5) \mathrm{Br}: \\
\text { material, } \\
\text { medicine, poison } \\
(3,11)\end{array}$ \\
\hline $\begin{array}{l}\text { Urticaceae } \\
\text { Girardinia } \\
\text { diversifolia (Link) } \\
\text { Friis }\end{array}$ & Allo sisnu & $\begin{array}{l}\text { Giant Asian } \\
\text { Nettles }\end{array}$ & Puuwâ & Herb & Rt & $\mathrm{Fd}$ & 1 & 0.32 & $\begin{array}{l}880 \mathrm{~J} \\
\text { KATH }\end{array}$ & $\begin{array}{l}\text { Bark is extracted for } \\
\text { fiber which is used to } \\
\text { make threads, ropes } \\
\text { and rough clothes. Root } \\
\text { decoction is applied on } \\
\text { sprains. }\end{array}$ & $\begin{array}{l}\text { Rt: medicine } \\
(1,2,10)\end{array}$ \\
\hline Botanical name & $\begin{array}{l}\text { Nepali } \\
\text { name }\end{array}$ & $\begin{array}{l}\text { English } \\
\text { name }\end{array}$ & $\begin{array}{l}\text { Local } \\
\text { name } \\
\text { (Magar) }\end{array}$ & $\begin{array}{l}\text { Life } \\
\text { form }\end{array}$ & $\begin{array}{l}\text { Parts } \\
\text { used }\end{array}$ & $\begin{array}{l}\text { Use } \\
\text { category }\end{array}$ & UV & UF & $\begin{array}{l}\text { Collection } \\
\text { number } I \\
\text { Herbarium }\end{array}$ & $\begin{array}{l}\text { Present findings } \\
\text { (indigenous uses) }\end{array}$ & $\begin{array}{l}\text { Previous } \\
\text { findings }\end{array}$ \\
\hline $\begin{array}{l}\text { Gonostegia hirta } \\
\text { (Blume ex Hassk.) } \\
\text { Miq. }\end{array}$ & $\begin{array}{l}\text { Chiple } \\
\text { Ghaans }\end{array}$ & $\begin{array}{l}\text { Glutious } \\
\text { rice-clumps }\end{array}$ & $\begin{array}{l}\text { Barmi } \\
\text { tinâ }\end{array}$ & Herb & Rt & Md & 1 & 0.12 & $t$ & $\begin{array}{l}\text { Root paste is applied to } \\
\text { treat bowls (Pilo). And, } \\
\text { also roots are eaten raw. }\end{array}$ & $\begin{array}{l}\text { Wp, Rt: medicine, } \\
\text { food, material } \\
(1,2,9,11)\end{array}$ \\
\hline Urtica dioica L. & Sisnu & $\begin{array}{l}\text { Stinging } \\
\text { nettle }\end{array}$ & Nganti & Herb & Lf & $\mathrm{Fd}$ & 1 & 0.32 & $\dagger$ & $\begin{array}{l}\text { Root decoction is used } \\
\text { to treat dandruff and } \\
\text { stomachache. Tender }\end{array}$ & Rt: medicine (1) \\
\hline
\end{tabular}


Ethnobotany Research and Applications

\begin{tabular}{|c|c|c|c|c|c|c|c|c|c|c|c|}
\hline & & & & & & & & & & \multicolumn{2}{|l|}{$\begin{array}{l}\text { shoots and leaves are } \\
\text { cooked as vegetable. }\end{array}$} \\
\hline $\begin{array}{l}\text { Vitaceae } \\
\text { Ampelocissus } \\
\text { rugosa (Wall.) } \\
\text { Planch. }\end{array}$ & & & Jiprang & $\begin{array}{l}\text { Climb } \\
\text { er }\end{array}$ & Lt & $\mathrm{Fd}$ & 1 & 0.14 & $\begin{array}{l}773 \\
\text { KATH/ } \\
\text { TUCH }\end{array}$ & $\begin{array}{l}\text { Ripe fruits are eaten } \\
\text { raw. Water content } \\
\text { (Sap) in internodes is } \\
\text { used to treat } \\
\text { conjunctivitis and latex is } \\
\text { used to treat warts } \\
\text { (Jojhai). }\end{array}$ & St: medicine (2) \\
\hline $\begin{array}{l}\text { Zingiberaceae } \\
\text { Acorus calamus } \mathrm{L} \text {. }\end{array}$ & Bojho & $\begin{array}{l}\text { Calamus } \\
\text { root }\end{array}$ & Baja & Herb & $\mathrm{Rh}$ & $\mathrm{Md}$ & 2 & 0.08 & $\dagger$ & $\begin{array}{l}\text { A small piece of rhizome } \\
\text { is chewed for cough or } \\
\text { sore throat. Rhizome } \\
\text { juice is used to treat } \\
\text { scabies. Necklace (Hâr) } \\
\text { is made for dog in case } \\
\text { of scabies (Khairâ). }\end{array}$ & $\begin{array}{l}\text { Rh: medicine } \\
(1,2,4,5,9,11)\end{array}$ \\
\hline $\begin{array}{l}\text { Curcuma } \\
\text { angustifolia Roxb. }\end{array}$ & $\begin{array}{l}\text { Barkhe } \\
\text { sarro }\end{array}$ & $\begin{array}{l}\text { East India } \\
\text { arrowroot }\end{array}$ & Kachur & Herb & Rt & $\mathrm{Md}$ & 1 & 0.14 & $\dagger$ & $\begin{array}{l}\text { Root paste is applied to } \\
\text { expedite the union of } \\
\text { fractured and dislocated } \\
\text { bones. }\end{array}$ & Rt: medicine $(2,9)$ \\
\hline $\begin{array}{l}\text { Hedychium } \\
\text { coronarium* }{ }^{*} \mathrm{~J} \text {. } \\
\text { Koenig }\end{array}$ & $\begin{array}{l}\text { Dudh } \\
\text { kewara }\end{array}$ & Garand lily & Tunti & Herb & Rt & $\mathrm{Md}$ & 1 & 0.04 & $\dagger$ & $\begin{array}{l}\text { Root paste is applied as } \\
\text { antidote for allergic } \\
\text { effect of } R \text { hus } \\
\text { succedeana. Beads } \\
\text { woven on rope are put } \\
\text { around child's neck to } \\
\text { relieve fever. }\end{array}$ & \\
\hline
\end{tabular}

Parts use: Rt: Root, Wd: Wood, St: Stem, Sh: Shoot, Br: Bark, Lf: Leaf, Fr: Fruit, Fl: Flower, Sd: Seed, Cu: Culm, Tu: Tuber, Rh: Rhizome, Rn: Resin, Ts: Tender shoot, Ct: Cotyledon, Wp: Whole Plant, Tr: Tree, Bu: Bulb, Lt: latex

Use categories: Dt: Dye and Tanning, Fb: fiber, Fd: Food, Fo: Fodder, FW: Fuelwood, Md: medicine, Po: Poison, SR: Social and Religious, Tm=Timber, Tr: Trade, Ol: Oil

Others: â: aa *: voucher specimen not available

Previous findings based on: 1-Baral and Kurmi (2006), 2- Manandhar (2002), 3- Ghimire et al. (2008), 4- Dutta (2007), 5-DPR (2007), 6- Bhattarai et al. (2006), 7- Lama et al. (2001), 8-Gautam

(2009), 9-Rajbhandari (2001), 10- Rajbhandary (2013), 11-Shrestha et al. (2017)

*New medicinal plants for Nepal 
Of all these MP species, 9 species cited by local Magar (Kham) people in the study area were reported as ethnomedicinally new to Nepal. The rest 73 species were found used both in the study area and elsewhere in other parts of the country. The species with new use reports are; Equisetum arvense, Cyperus cyperoides, Hedychium coronarium, Clematis terniflora, Delphinium grandiflorum, Rubus hoffmeisterianus, Geranium procurrens, Cornus capitata, and Taraxacum parvulum. MP species like Aconitum gammiei not found in the study area, brought from other parts of Nepal has also been used by the local people to treat disease since long before. Among total MP species, 44 species were used only for medicinal purpose whereas 38 species were used for multi-purposes besides medicine. Of the total plant species with multiple uses; 20 MP species were used for medicine and food, followed by medicine and socio-religious (5spp.), medicine and timber (4 spp.), medicine and dye (4 spp.), and medicine and fiber (3). Likewise, 3 MP species were used for medicine, food and socioreligious and 3 species for medicine, food and poison (Figure 2). While comparing the families that contributed more medicinal species was Ranunculaceae represented by 8 species $(9.75 \%)$ followed by Fabaceae and Rosaceae with 6 species each (7.32\%), Polygonaceae with 4 species (4.88\%), Orchidaceae, Urticaceae and Zingiberaceae with 3 species each $(3.66 \%)$ where other 38 families were with 49 species $(59.76 \%)$ represented by 1 or 2 species. Of the total species, $5 \%$ were traded.

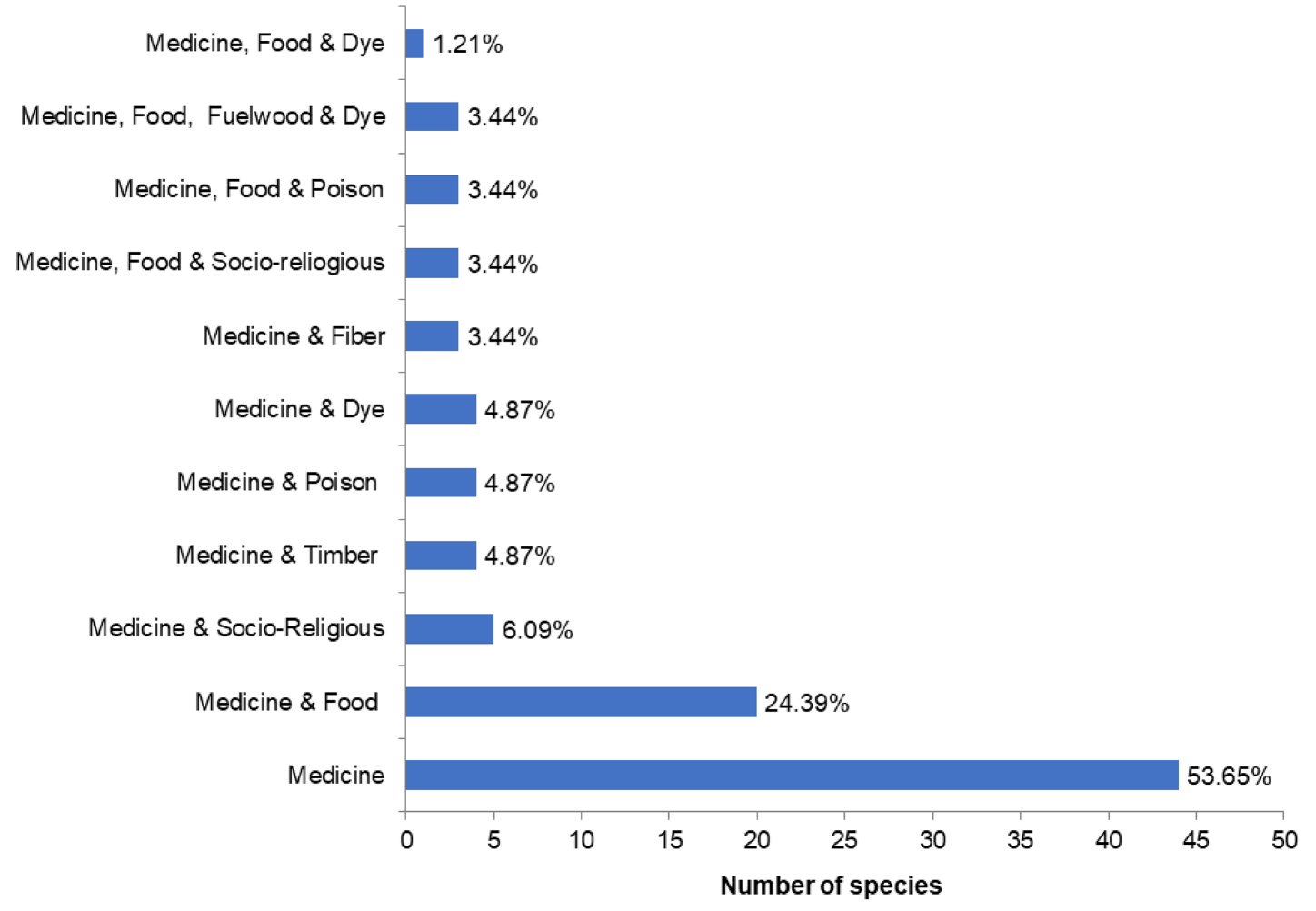

Figure 2. Number of MP species with single and multiple uses.

In FGD, there were 95 participants whereas in individual there were 50 . Out of 95 participants in FGD, there were more male $(n=55)$ compared to female $(n=40)$. However, among the 50 interviewees in individual interview, majority of them were female $(n=30)$ and rest $(n=20)$ were male where 5 were Jhankries; the traditional healers. Mostly all informants (38) were Magar (Kham) people belonging to different sub-castes (Roka, Budha, Gharti and Jhankri) and 12 were occupational groups (Nepali and Bishowkarma). While by occupation $90 \%$ of the people were involved in agriculture and animal husbandry. Only $5 \%$ were engaged in business and services like teaching whereas rest $5 \%$ were in other occupation. Regarding their level of knowledge on use of MP species, the traditional healers were more knowledgeable compared to other local people. Among the interviewee, male and old participants had more knowledge than female on use of MP species.

Life forms, plant parts used, method of preparation and administration

Majority of MP species were herbs $(67 \%, n=55)$, followed by trees $(16 \%, n=13)$, shrubs $(13 \%, n=11)$ and climbers $(4 \%, n=3)$. Altogether, 8 different parts of these plants were used. Most of these species were harvested for root $(36.58 \%)$, followed by leaves $(14.63 \%)$, bark $(9.75 \%)$, fruits $(6.09 \%)$ and the 
whole plant in case of herb species (12.19\%) (Figure 3 ). According to local people, there are certain MP species that should be collected on specific time for their medicinal potentiality. For instance, Zanthoxylum armatum and Lindera neesiana must be collected on $12^{\text {th }}$ of Bhadra (28th August). Since, they have faith that fruits collected on that particular date have effective medicinal property. On the other hand, they use MP species in mixed form. They use species such as Allium sativum, Potentilla spp., Plantago asiatica, Quercus semecarpifolia and Prunus cerasoides in mixed form. In some cases, species like Allium sativum was mixed with dried human placenta and used as an antidote for cattle. Barks of Quercus semicarpifolia, Quercus lanata and Prunus cerasoides were cooked together, thus the decoction obtained was used to get relief from sprains. As far as the mode of preparation was concerned, majority of MP species were used in the form of mastication (21) followed by juice (16), paste (15) and decoction (13) (Figure. 4). In some cases, sap, latex and resins of the plants were also used as an ointment. A decoction was prepared by boiling the plant in water until reduced to the half volume or less than the original volume of the liquid. The juice was obtained by squashing leaf or whole plant. The paste was prepared by grinding plant parts with water. Tea was prepared by just boiling the plant part in water.
On mode of admission, the most common method was oral $(51 \%, n=42)$, followed by external or topical application $(47 \%, n=39)$ and inhalation $(2 \%, n=2)$.

Ailments treated in the study area

Medicinal plants were used to treat 32 ailments in the study area. With regard to ailments, the highest number of medicinal plants (20 spp.) were prescribed for the stomachache followed by external wounds (burns, cuts \& wounds) (19 species), diarrhea (17 species), gastritis and fever (10 species each), arthritis (9 species) and sprains (8 species) (Table 1).

\section{Informant consensus factor and important values}

The results of informant consensus factor (Fic) calculation showed that the Fic values ranged from 0 to 1. Dandruffs, cancer, frightening, warts, pneumonia, jaundice, skin and eye problem ailments had the highest Fic value 1 with 2, 3 or 4 use reports for single species. The second highest Fic value obtained was for cough ailments with 26 use-reports for 6 species followed by gastritis ( $F i c=0.7$, UR $=31$ and $10 \mathrm{spp}$.). The least Fic value was observed for typhoid with 4 use-reports and for 4 species, scabies with 3 use-reports and 3 species and toothache with 2 use reports and 2 species (Table 2 ).

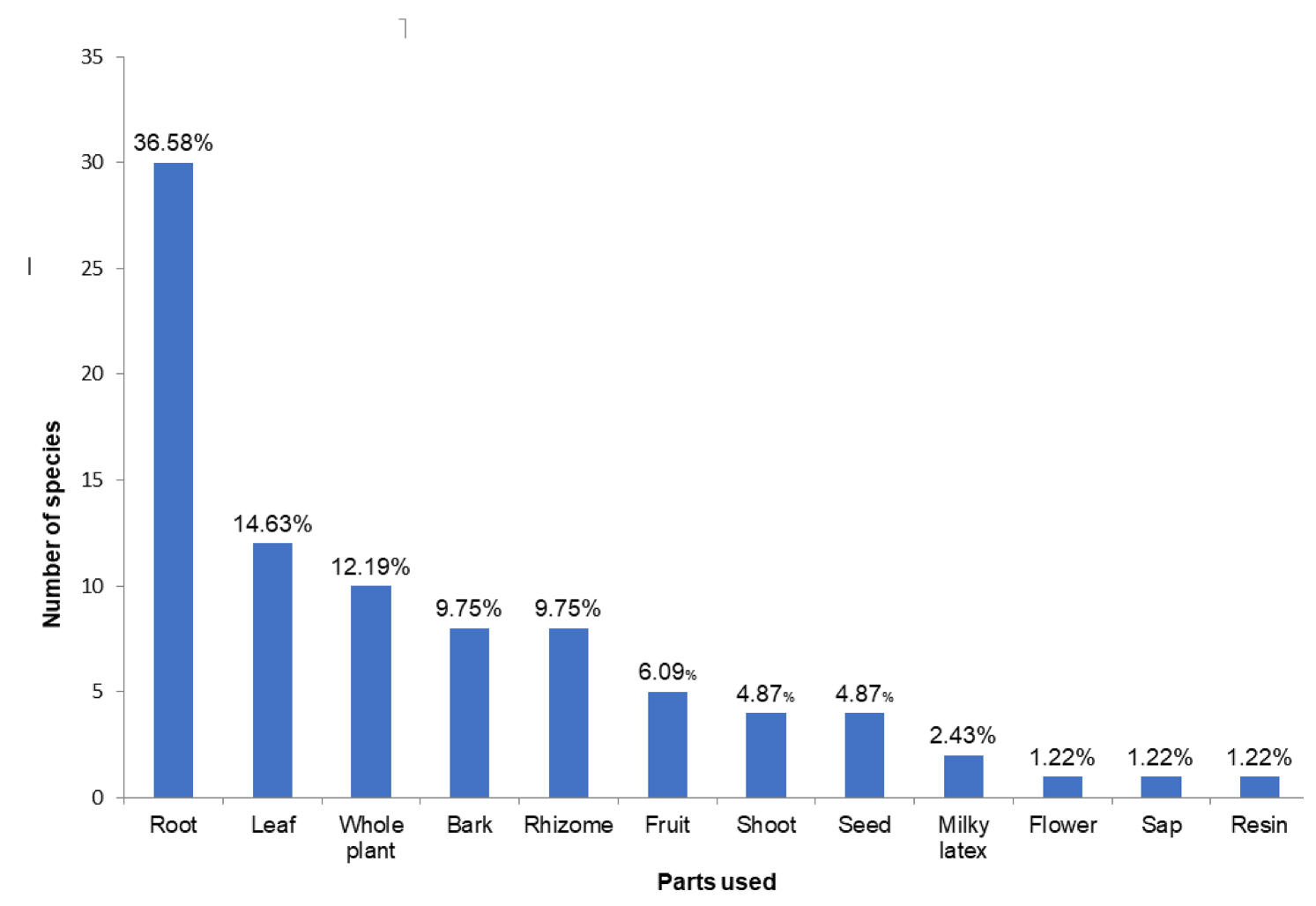

Figure 3. Plant parts used based on field survey. 


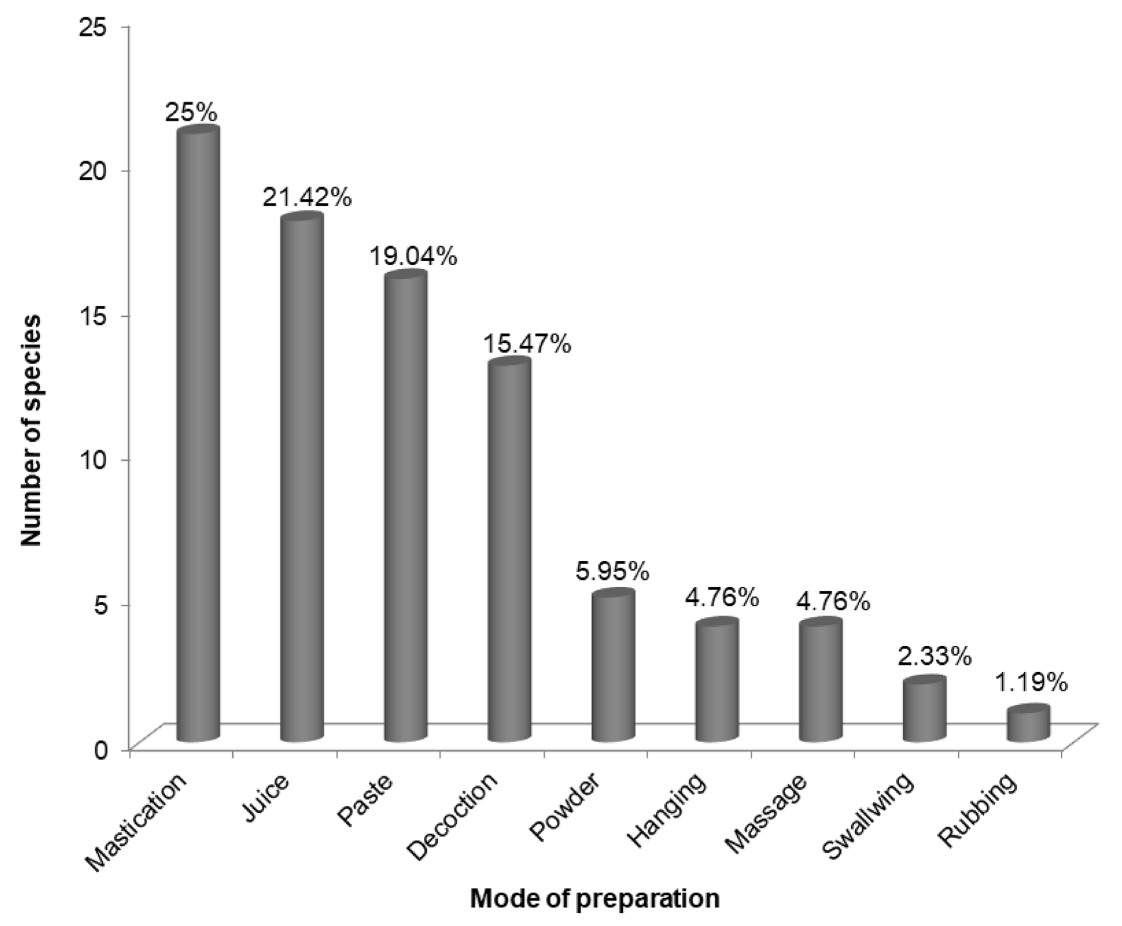

Figure 4. Number of plant species used in different mode for medicinal purpose

Table 2. Informant consensus factor (Fic) for different ailment categories.

\begin{tabular}{|c|c|c|c|c|}
\hline Ailments & $\begin{array}{l}\text { Number } \\
\text { of Taxa } \\
\text { (Nt) }\end{array}$ & $\begin{array}{l}\text { Number of } \\
\text { use reports } \\
\text { (Nur) }\end{array}$ & IFC & Name of species \\
\hline Stomachache & 20 & 31 & 0.37 & $\begin{array}{l}\text { Aconitum gammiei, Aconitum spicatum, Bergenia } \\
\text { ciliata, Brucea javanica, Dactylorhiza hatagirea, } \\
\text { Delphinium grandiflorum, Delphinium vestitum, } \\
\text { Fagopyrum megacarpum, Flemingia strobilifera, } \\
\text { Heracleum candicans var. obtusifolium, Leibnitzia } \\
\text { nepalensis, Paris polyphylla, Potentilla lineata, } \\
\text { Quercus semecarpifolia, Rheum australe, } \\
\text { Rhododendron campanulatum, and Rosa macrophylla, } \\
\text { Satyrium nepalense, Solena heterophylla, Urtica } \\
\text { dioica, Zanthoxylum armatum }\end{array}$ \\
\hline $\begin{array}{l}\text { Burns, Cuts \& } \\
\text { Wounds }\end{array}$ & 19 & 72 & 0.75 & $\begin{array}{l}\text { Aconitum spicatum, Ageratina adenophora, Anemone } \\
\text { vitifolia, Cheilanthes albomarginata, Delphinium } \\
\text { vestitum, Dipsacus inermis, Drymaria cordata, Galium } \\
\text { asperuloides, Leibnitzia nepalensis, Lindenbergia } \\
\text { muraria, Myriactis nepalensis, Paris polyphylla, Pinus } \\
\text { wallichiana, Bistorta milleti, Rubia manjith, Selaginella } \\
\text { biformi, Stellaria himalayensis, Swertia chirayta, and } \\
\text { Taraxacum parvulum }\end{array}$ \\
\hline Diarrhea & 17 & 40 & 0.59 & $\begin{array}{l}\text { Ageratina adenophora, Bergenia ciliata, Brucea } \\
\text { javanica, Campylotropis speciosa subsp. Eriocarpa, } \\
\text { Cornus capitate, Daphne bholua, Equisetum arvense, } \\
\text { Fagopyrum megacarpum, Flemingia strobilifera, } \\
\text { Imperata cylindrical, Leucosceptrum canum, Plantago } \\
\text { asiatica subsp. erosa, Potentilla lineata, Rheum } \\
\text { austral, Rumex nepalensis, Swertia chirayta and } \\
\text { Verbascum thapsus }\end{array}$ \\
\hline Gastritis & 10 & 31 & 0.70 & $\begin{array}{l}\text { Bergenia ciliata, Myriactis nepalensis, Fagopyrum } \\
\text { megacarpum, Cyperus cyperioides, Plantago asiatica } \\
\text { subsp. erosa, Potentilla lineata, Rubus } \\
\text { hoffmeisterianus, Rumex nepalensis, Zanthoxylum } \\
\text { armatum, and Phytolacca latbenia }\end{array}$ \\
\hline
\end{tabular}




\begin{tabular}{|c|c|c|c|c|}
\hline Ailments & $\begin{array}{l}\text { Number } \\
\text { of Taxa } \\
\text { (Nt) }\end{array}$ & $\begin{array}{l}\text { Number of } \\
\text { use reports } \\
\text { (Nur) }\end{array}$ & IFC & Name of species \\
\hline Fever & 10 & 12 & 0.18 & $\begin{array}{l}\text { Aconitum gammiei, Bergenia ciliata, Cirsium verutum, } \\
\text { Cynodon dactylon, Daphne bholua, Paris polyphylla, } \\
\text { Taraxacum parvulum, Verbascum thapsus, Morina } \\
\text { longifolia, and Hedychium coronarium }\end{array}$ \\
\hline Arthritis & 9 & 18 & 0.53 & $\begin{array}{l}\text { Anemone rivularis, Arisaema jacquemontii, Cannabis } \\
\text { sativa, Clematis terniflora, Agave Americana, Swertia } \\
\text { chirayta, and Curcuma angustifolia }\end{array}$ \\
\hline Antidote & 8 & 24 & 0.70 & $\begin{array}{l}\text { Aconitum gammiei, Allium sativum, Asparagus } \\
\text { racemosus, Lindera neesiana, Solena heterophylla, } \\
\text { Delphinium vestitum, Paris polyphylla, and } \\
\text { Zanthoxylum armatum }\end{array}$ \\
\hline Sprain & 8 & 22 & 0.67 & $\begin{array}{l}\text { Cerasus cerasoides, Geranium procurrens, Girardinia } \\
\text { diversifolia, Prunella vulgaris, Quercus lanata, Q. } \\
\text { semecarpifolia, Rheum australe, and Rumex } \\
\text { nepalensis }\end{array}$ \\
\hline Cough & 6 & 26 & 0.80 & $\begin{array}{l}\text { Acorus calamus, Bergenia ciliata, Lindera neesiana, } \\
\text { Rhododendron arboreum, Swertia chirayta, and } \\
\text { Zanthoxylum armatum }\end{array}$ \\
\hline Bone fracture & 5 & 9 & 0.50 & $\begin{array}{l}\text { Allium tuberosum, Pleione humilis, Ageratina } \\
\text { adenophora, Aconitum gammiei, and Viscum album }\end{array}$ \\
\hline Headache & 5 & 7 & 0.33 & Dactylorhiza hatagirea, and Swertia chirayta \\
\hline Worms & 5 & 6 & 0.20 & $\begin{array}{l}\text { Acorus calamus, Arisaema jacquemontii, Artemisia } \\
\text { dubia, Imperata cylindrica, and Rubia manjith }\end{array}$ \\
\hline Allergy & 4 & 5 & 0.25 & $\begin{array}{l}\text { Aconitum gammiei, Acorus calamus, Rumex } \\
\text { nepalensis, and Hedychium coronarium }\end{array}$ \\
\hline Delivery & 4 & 5 & 0.25 & $\begin{array}{l}\text { Lindera neesiana, Delphinium vestitum, Paris } \\
\text { polyphylla, and Zanthoxylum armatum }\end{array}$ \\
\hline Typhoid & 4 & 4 & 0.00 & $\begin{array}{l}\text { Aconitum gammiei, Dactylorhiza hatagirea, Swertia } \\
\text { chirayta, and Delphinium grandiflorum }\end{array}$ \\
\hline Heart Pain & 3 & 5 & 0.50 & $\begin{array}{l}\text { Aconitum gammiei, Allium sativum, and Rosa } \\
\text { macrophylla }\end{array}$ \\
\hline Mumps & 3 & 4 & 0.33 & Verbascum thapsus, Gonostegia hirta, Aesculus indica \\
\hline Scabies & 3 & 3 & 0.00 & $\begin{array}{l}\text { Acorus calamus, Rhododendron campanulatum, and } \\
\text { Rumex nepalensis }\end{array}$ \\
\hline Jaundice & 2 & 8 & 0.86 & Berberis asiatica, and Swertia chirayta \\
\hline Vomiting & 2 & 3 & 0.50 & Bergenia ciliata, and Swertia chirayta \\
\hline Asthma/ Dam & 2 & 3 & 0.50 & Cotoneaster microphyllus, and Rheum australe \\
\hline Itching & 2 & 3 & 0.50 & Acorus calamus, and Clematis terniflora \\
\hline Toothache & 2 & 2 & 0.00 & Juglans regia, and Begonia picta \\
\hline Dandruffs & 1 & 3 & 1.00 & Urtica dioica \\
\hline Cancer & 1 & 2 & 1.00 & Tsuga dumosa \\
\hline Frightening & 1 & 2 & 1.00 & Equisetum arvense \\
\hline Warts & 1 & 5 & 1.00 & Ampelocissus rugosa \\
\hline Pneumonia & 1 & 3 & 1.00 & Cannabis sativa \\
\hline Anesthetic & 1 & 7 & 1.00 & Swertia chirayta \\
\hline Skin fractures & 1 & 2 & 1.00 & Pinus wallichiana \\
\hline Eye irritation & 1 & 5 & 1.00 & Ampelocissus rugosa \\
\hline Leech & 1 & 4 & 1.00 & Anemone rivularis \\
\hline
\end{tabular}

The most frequently used species reported in the study area were Berberis aristata and Swertia chirayta (UF=0.48 each) with 24 informants among the total informants interviewed. The second most frequently used species was Bergenia ciliata (UF=0.42) with 21 informants followed by Cerasus cerasoides (UF=0.38, n=19), Ageratina adenophora $(\mathrm{UF}=0.36, \mathrm{n}=18)$ and Anemone vitifolia $(\mathrm{UF}=0.34$, $\mathrm{n}=18$ ). Similarly, the plant species with highest use value (UV) was Bergenia ciliata (3.29) followed by Aesculus indica (2.75), Berberis aristata (2.42), Swertia chirayta (2.08), Acorus calamus (2.00), Arisaema jacquemontii (2.00), Delphinium vestitum
(1.93), Cyperus cyperiodes (1.75), Dactylorhiza hatagirea (1.67), Taraxacum parvulum (1.60) and Lindera neesiana (1.57) (Table 1).

Threats to MP species and their conservation During FGD, informants deciphered unsustainable harvesting of NTFPs (non-timber forest products) including premature collection of MP species from wild for swift monetary gain, lack of forest management system, deforestation and increasing population are the main threats to MP species in the study area. They were with single voice to save important MP species for their future generation. 
Similarly, most informants (95\%) of individual interview confirmed that MP species are collected from wild for both medicinal uses and trade. Moreover, traditional healers were reluctant to share their knowledge. $10 \%$ of the informants were cultivating few traded MP species in their home gardens. The species included Valeriana hardwickii, Bergenia ciliata, Paris polyphylla, and Rheum australe. However, all informants were aware for the conservation of the MP species.

\section{Discussion}

\section{Medicinal Plant and their indigenous use}

This very first ethno-medicinal research carried out among the Magar (Kham) people identified 82 MP species from Thabang RM in Jaljala region, of which 9 MP species were identified as new use report to Nepal. These nine species were used differently in treatment of various disorders (Additional file 2) while comparing to previous ethnobotanical studies in Nepal himalaya (Lama et al. 2001, Rajbhandari 2001,2013, Manandhar 2002, Baral \& Kurmi 2006, Bhattarai \& Ghimire 2006, DPR 2007, Dutta 2007, Ghimire \& Pyakurel 2008, Rokaya et al. 2010, Shrestha et al. 2018). For instance, root and stem of Equisetum arvense were mixed with algae and Rumex nepalensis. during fever, root of Cyperus cyperoides were used in case of gastritis, root of Hedychium coronarium as antidote in allergies of Rhus succedeana, aerial parts of Clematis terniflora in gout, root of Delphinium grandiflorum in stomachache, leaf juice of Rubus hoffmeisterianus in in-appetite, root of Geranium procurrens mixed with other plants (such as Girardiana diversifolia, Rheum austral, Rumex nepalensis. and Prunus cerasoides), ripe fruit of Cornus capitata in headache, and leaf juice or latex of Taraxacum parvulum in healing cuts and wounds. Furthermore, there was trend of using MP species such as Aconitum gammiei which is not found in the study area to treat stomachache since long before, typically in families of sheepherders. Since, the sheepherders collect the roots of Aconitum gammiei from high altitude during their summer grazing season and store for the future use.

Out of 82 species, most of MP species (44 spp.) were used only for medicinal purpose and rest 38 MP species had multi-purposes, such as food, socioreligious, fibber, fodder and dye in addition to their medicinal uses. In general, MP species with multiple use purposes are more threatened rather than single use. So, these species should be prioritized for cultivation, conservation and management for their sustainability (Geng et al. 2017, Napagoda et al. 2018, Kidane et al. 2018). Additionally, they should be considered as an ideal resource for management tenure for future income generation of forest dwelling people and wildlife (Cummings 2013). While comparing the families, Ranunculaceae was the most frequently used family. Since, it is one of the largest families among the vascular plant species and most species of this family contain high biochemical compounds with anti-inflammatory and antioxidant properties (Malik et al. 2017).

Regarding the livelihood activities, most of Magar (Kham) people were engaged in agriculture and animal husbandry. So, the most of them were dependent on plant resources mainly on MP for their basic primary health care specifically in cuts and wounds, gastro-intestinal disorders and sometimes in case of eye irritation too. Furthermore, the tendency of use may be because of their traditional practice which has been more culturally acceptable and practiced since time immemorial (SaslisLagoudakis et al. 2014). The older people were more knowledgeable but trend of passing their knowledge was found to be declining.

Life forms, plant parts used, method of preparation and administration

Regarding the collection and utilization of MP species, local Magar (Kham) people have quite specialized knowledge. Among different life forms, herbs were most commonly used species where local people harvest mainly their roots (similar to previous studies e.g. Baral \& Kurmi 2006, Rokaya et al. 2010, Kunwar et al. 2006). This may be because herbs are more abundant, easy to collect and roots contain high concentration of bioactive compounds compared to other parts (Srithi et al. 2009). But, the removal of whole plant or roots or excessive use of herbs for medicine lead to the decline in population of the MP species (Ghimire \& Pyakurel 2008). The removal of underground part is much more unsustainable compared to aerial part (Kunwar et al. 2006). But only few species have been harvested for their aerial parts such as fruits, leaves and barks in the study area. Concerned with plant collection time and condition, Magar (Kham) people are quite specific in harvesting MP species as Gurungs of Kaski, Central Nepal (KC et al. 2010). They harvest according to different seasons, climatic conditions and habitat types for treatment of ailments similar to the Yao people in China (Long \& Li 2004). For example, informants reported that the fruits of Zanthoxylum armatum and Lindera neesiana should be collected on particular date. This is supposed to be true because, these plants contain highly volatile compounds (Ivanova et al. 2004) which are altered by multiple parameters; seasonal variations, degree of maturity of plant atmosphere, precipitation, or geographic location of the plant (Marotti et al.1994, Hussain et al. 2008, Anwar et al. 2009). 
Regarding mode of preparation, they followed different techniques for various MP species. They were quite specific for way of using. For example, they know how much amount and in which form to apply and administer for particular ailment categories such as stomachache, external wounds, breathing problems and excessive physical works (Manandhar 2002). Generally, they used MP species singly whereas they use species such as Plantago asiatica, Potentilla lineata, Allium sativum, Equisetum arvense and Verbascum thapsus in mixed form either with 2, 3 or 4 species. This multiple preparation methods were based on the knowledge provided to the individual by their predecessor (Uprety et al. 2012, Bhattarai et al. 2006). They follow simple techniques while using MP species. Hence, majority of plant species were used simply just after mastication. In case of gastro-intestinal disorders like stomachache, gastritis, heartburn and jaundice, masticated form of MP species was used. In some cases, like cuts, wounds and burns, juice and paste of the plant were applied. In sprains and some gastrointestinal disorders, decoction prepared by distillation of plant parts was used. Regarding, mode of administration MP species were commonly used orally which was followed by topical i.e. an external application and then inhalation (similar to Shrestha \& Dillion 2003, Bhattarai et al. 2006, Luitel et al. 2014, Singh et al. 2012). According to informants, antidotes are diluted in case of adverse effects either by minimizing size of use parts or mixed with water. For instance, minimal powder of Paris polyphylla was used on wounds, small piece of roots of Bergenia ciliata, and Aconitum gammiei during stomachache. Furthermore, they were specifically knowledgeable on the use of plant parts. We found that they use various parts of single species in different use categories. For example, from the single species Arisaema jacquemontii they use fresh seeds against worm infestation as medicine, leaves cooked with Rumex hastatus as vegetable and fresh corms as poison. Thus, the area is medicinally biodiverse and hence there is ethnodiverse on use of plants (Begossi 1996).

The highest number of MP (20) species were prescribed for stomachache. This may be because, these species were easily available and their extensive ethnomedicinal usage become an important component in their medical system and hence their part of culture too (Tardio \& Santayana 2008, Kunwar et al. 2014)

\section{Cultural values of MP species}

To understand the cultural values of MP species, quantitative techniques; Informant Consensus Factor (Fic), Use Frequency (UF) and Use Value (UV) are important parts of ethnobotany. Fic values were determined in order to know the agreement among the informants of the study area for the use of plants to treat certain ailment categories. The Fic value in 8 ailment categories was found '1' (Table 2). This indicated a high level of informant agreement compared to similar studies conducted in Nepal Himalaya (Kunwar et al. 2010). The plant species having high value of Fic were supposed to be efficient in treating particular ailment. In this study Urtica dioica with $\mathrm{Fic}=1$ may be sufficient in treatment of dandruffs, similarly Ampelocissus rugosa in treatment of warts, Taxus contorta in treatment of cancer and Swertia chirayta in treatment of jaundice. So, the plant species that have high consensus value are of socio-economically significant and important for further pharmacological research (Rossato et al. 1999, Canales et al. 2005, Hong et al. 2015).

The UF of MP species Berberis aristata, Swertia chirayta, Bergenia ciliata, Prunus cerasoides, Ageratina adenophora and Anemone vitifolia was high. This is because these MP species are effective to use and easy for accessibility during collection. Similarly, the high UV obtained for Bergenia ciliata, Asesculus indica, Berberis aristata, Swertia chirayta, Acorus calamus, Arisaema jacquemontii, Delphinium vestitum, Cyperus cyperoides, Dactylorhiza hatagirea, Taraxacum parvulum and Lindera neesiana. Since, informants reported that these MP species are used in multiple number of ailment categories. Thus, the highest important (Fic, UF \& UV) values shown by these MP species indicate that these species are highly preferred. Furthermore, this shows that these species are contextually, culturally more important and theoretically they have high cultural consensus (Tardio \& Santayana 2008). Additionally, they may have high phytochemical constituents. Therefore, those species must be tested further for their phytochemical property (Akgul et al. 2018).

\section{Medicinal plant cultivation, trade, threats and conservation practices}

The Magar (Kham) people of the study area have the knowledge on the benefits of conserving the medicinal plants. However, the effort of conserving MP species was hard because mostly all of the MP species were collected from wild. Even the traditional healers (Jhankries) who most frequently use did not practice cultivating common MP species in their home garden, they preferred to harvest from wild. Since, they wanted to have secrecy over their use of MP species and worry about their occupation (Bhattarai et al. 2006). Therefore, they were unwilling to share their knowledge of using MP species they use with local villagers. Few MP species like Valeriana jatamansii, Bergenia ciliata, Paris 
polyphylla, and Rheum australe were locally traded from study area. Therefore, recently there is increasing interest for cultivating the MP species among the local people. Many of them are in search of possibility of MP species cultivation.

Various factors that were considered as main threats for medicinal plants were recorded during discussion with the informants in the study area. The major threats of MP species include lack of forest management system, pressure from anthropogenic activities such as deforestation, unsustainable harvesting of NTFPs (non-timber forest products) including premature collection of MP species from wild. Moreover, the population expanding throughout has accelerated the expansion of the agricultural land, urbanization, overharvesting of MP species and fuelwood collection. On discussion with local people, they suggested that there should be the establishment of community forest, initiation of cultivation NTFPs and enforce strict law for the conservation of MP as well as other useful plant species.

\section{Reliability of reported uses}

The indigenous uses of plants obtained in present study were compared with the published information from other parts of Nepal (e.g. Lama et al. 2001, Manandhar 2002, Baral \& Kurmi 2006, Rajbhandari 2001, 2013, Bhattarai \& Ghimire 2006, Dutta 2007, DPR 2007, Ghimire \& Pyakurel 2008, Rokaya et al. 2010). Comparing with these available literatures, 73 ethnomedicinal species used in the study area were similar to uses in other parts of the country but 9 ethnomedicinal species which are indicated by asterisk $\left({ }^{*}\right)$ were reported new for Nepal (Table 1$)$.

\section{Conclusions}

This very first ethnomedicinal study carried out in Magar (Kham) people added nine species in the list of medicinal plants to the country. The new reported species were Equisetum arvense, Cyperus cyperoides, Hedychium coronarium, Clematis terniflora, Delphinium grandiflorum, Rubus hoffmeisterianus, Geranium procurrens, Cornus capitata, and Taraxacum parvulum. Thus, there is furthermore possibility of recording new use species through extensive ethnobotanical study in adjoining villages of present study area. Magar Kham people are highly knowledgeable, specifically in the use of medicinal plant, so high diversity of medicinal species has been reported. Quantitative ethnobotanical analysis revealed that 82 species were used for various ailment categories. Species such as Berberis aristata, Swertia chirayta, Bergenia ciliata, Cerasus cerasoides, Ageratina adenophora and Anemone vitifolia were frequently used species and species such as Bergenia ciliata, Aesculus indica, Berberis aristata, Swertia chirayta, Acorus calamus, Arisaema jacquemontii, Delphinium vestitum, Cyperus cyperodes, Dactylorhiza hatagirea, Taraxacum parvulum and Lindera neesiana were most valuable species based on use value. The highest important (Fic, UF \& UV) values shown by medicinal plants indicated that these species were highly preferred by local people which might be due to their effectiveness because of high phytochemical constituents. So, these MP species with high important values are highly recommended for further pharmacological tests.

\section{Declarations}

Lista de Abreviaturas: No aplica

Ethics approval and consent to participate: Permission letters were taken from Department of Forest and Soil Conservation, Babarmahal, Kathmandu prior to data collections. Oral agreements were obtained from the local informants about the aims and objectives of the study prior to interviews and all field data were collected through their oral consents. No further ethics approval was required.

Consent for publication: This paper does not include any individual person's data and further consent for publication is not required.

Availability of data and materials: The data are available from the autjors upon request.

Competing interests: The authors declare that they have no competing interest.

Funding: As this research study is the part of first author's master's dissertation work, so she managed herself. We did not have any other sources of funding.

Authors' contribution: SBM and PB carried out field work, collected data and identified Taxa. SBM analyzed data and prepared the draft for the manuscript. PB made map of study area and edited the manuscript. SKG supervised the project.

\section{Acknowledgements}

This research is part of the Master's research work of the principal author. The authors would like to thank traditional healers and local people of Thabang for sharing their knowledge and kind cooperation and hospitality during field. Furthermore, Kendall Mormann, M.Sc. is accredited for language correction.

\section{Literature cited}

Acharya R. 2012. Ethnobotanical study of medicinal plants of Resunga Hill used by Magar community of Badagaun VDC, Gulmi district, Nepal. Scientific World 10(10): 54-65.

Albuquerque UP, Silva JS, Campos JLA, Sousa RS, Silva TC, Alves RRN. 2013. The current status of 
ethnobiological research in Latin America: gaps and perspectives. Journal of Ethnobiology Ethnomedicine 9:72.

Ale R, Raskoti BB, Shrestha K. 2009. Ethnobotanical knowledge of Magar community in Siluwa VDC, Palpa district, Nepal. Journal of Natural History Museum 24(1): 58-71.

Alexiades MN. 2003. Ethnobotany in the third Millennium: expectations and unresolved issues. Delpinoa 45:15-28.

Akgul A, Akgul A, Senol SG, Yildirim, Secmen O, Dogan Y. 2018. An ethnobotanical study in Midyat (Turkey), a city on the silk road where cultures meet. Journal of Ethnobiology Ethnomedicine 14:12.

Anwar F, Hussain Al, Sherazi STH, Bhanger MI. 2009. Changes in composition and antioxidant and antimicrobial activities of essential oil of fennel (Foeniculum vulgare Mill.) fruit at different stages of maturity. Journal of Herbs, Spices and Medicinal Plants. 15(2): 187-202.

Arulappan MT, Britto SJ, Ruckmani K, Mohan R. 2015. An ethnobotanical study of medicinal plants used by ethnic people in Gingee Hills, Villupuram District, Tamilnadu, India. American Journal Ethnomedicine 2(2): 2348-9502.

Balaraju S, Ramamurthy N, Konkala A, Suresh S. 2015. Ethnomedicinal plants used to cure skin diseases by tribals of Mahabubnagar district, Telangana state. IOSR Journal of Pharmacy and Biological Scicences (IOSR-JPBS) 10(6): 25-27.

Baral SR, Kurmi PP. 2006. A Compendium of Medicinal Plants in, Mass Printing Press Kathmandu, Nepal.

Bennett BB. 1996. A discipline in search of a definition. Book Review of Ethnobotany: Evolution of a Discipline. R.E. Schultes and S. Von Reis. 1994. Timber Press, Bio Science Portland, USA.

Bhattarai KR, Ghimire M. 2006. Commercially important medicinal and aromatic plants of Nepal and their distribution pattern and conservation measure along the elevation gradient of the Himalayas. Banko Jankari 16: 3-13.

Bhattarai S, Chaudhary RP, Taylor RSL. 2006. Ethnomedicinal plants used by the people of Manang district, central Nepal. Journal of Ethnobiology and Ethnomedicine 2:41.

Bigossi A. 1996. Uses of ecological methods in ethnobotany: diversity indices. Economic Botany 50(3):280-289.

Budha-Magar S. 2020. Ethnic uses of plant species among the Magar people in Western Nepal. In Wild
Plants: The Treasure of Natural Healers. Edited by Rai M and Bhattarai S, CRC Press, USA (in press).

Byg A, Baslev H. 2001. Diversity and use of palms in Zahamena, eastern Madagascar. Biodiversity Conservation 10(6):951-970.

Canales M, Hernandez T, Caballero, Romo de Vivar A, Avila G, Duran A, Lira R. 2005. Informant consensus factor and antibacterial activity of the medicinal plants used by the people of San Rafael Coxcatlan, Puebla, Mexico. Journal of Ethnopharmacology 97:429-439.

CBS [Central Bureau of Statistics]. 2011. Nepal Census 2011. District profiles (Demography). District Profile of Rolpa. Central Bureau of Statistics (CBS), National Planning Commission Secretariat, Kathmandu, Nepal. (Demography). Accessed from http://ddcrolpa.gov.np/wp-

content/uploads/2016/05/शंक्षिप्त-परिचय.pdf on April 16, 2019

Cummings AR. 2013. For logs, for traditional purposes and for food: identification of multiple-use plant species of northern Amazonia and an assessment of factors associated with their distribution. Dissertations - ALL. 17. https://surface.syr.edu/etd/17.

Cunningham AB, Shanley P, Laird S. 2008. Health, habitats and medicinal plant use. In: Colfer C.J.P., editor. Human Health and Forests: A Global Overview of Issues, Practice and Policy. Earthscan; London, UK.

Davis EW. 1995. Ethnobotany: an old practice, a new discipline. In ethnobotany: Evolution of a Discipline (R.E. Schultts and S.V. Reis, edition). Dioscoriodes Press, Oregon.

De Boer HJ, Cotingting C. 2014. Medicinal plants for women's healthcare in southeast Asia: a metaanalysis of their traditional use, chemical constituents, and pharmacology. Journal of Ethnopharmacology 151(2): 747-767.

DPR [Department of Plant Resources]. 2007. Bulletin of the Department of Plant Resources No. 28 Medicinal Plants of Nepal (Revised). Department of Plant Resources, Ministry of Forest and Soil Conservation, Government of Nepal, Thapathali, Kathmandu, Nepal .

Dutta IC. 2007. Non-timber forest products of Nepal: identification, classification, ethnic uses and cultivation. Institute of Forestry, Pokhara, Nepal.

Ford RL. 1978. The nature and status of ethnobotany. In Anthropological Papers (R.L. Ford, ed.), Museum of Anthropology, University of Michigan, USA. 
Forman L, Bridson D. 1989. The Herbarium Handbook. Royal Botanic Gardens Kew, Great Britain.

Geng Y, Hu G, Ranjitkar S, Wang Y, Bu Denpan, Pei S, Ou X, Lu Y, Ma X, Xu J. 2017. Prioritizing fodder species based on traditional knowledge: a case study of mithun (Bos frontalis) in Dulongjiang area, Yunnan Province, Southwest China. Journal of Ethnobiology and Ethnomedicine 13:24.

Ghimire SK, Shrestha AK, Shrestha KK, Jha PK. 2000. Plant resources use and human impact around Royal Bardiya National Park, Nepal. Journal of Natural History Museum (Kathmandu) 19:3-26.

Ghimire SK, Pyakurel D. 2008. A Manual of NTFs of Nepal Himalaya (Gair Kastha Ban Paidawar Digdarshan), Kathmandu, Nepal. WWF Nepal.

Grierson AJ, Long DG. 1983. Flora of Bhutan, including a record of plants from Sikkim. Vol. 1, Part 1 and 2. Royal Botanic Garden.

Hara H, Stearn WT, Williams LHJ. 1978. An enumeration of the flowering plants of Nepal; a joint project of the British Museum (Natural History) and the Univ. of Tokyo-v. 1: (Gymnospermae and Angiospermae (Monocotyledones)), British Museum of Natural History, London.

Hara H, Stearn WT, Williams LHJ. 1982. An enumeration of the flowering plants of Nepal. British Museum (Natural History), London.

Hara H, Williams LHJ. 1979. An enumeration of the flowering plants of Nepal. British Museum of Natural History, London.

Harshberger JW. 1896. The purpose of Ethnobotany. Bot Gaz. 21: 146-154.

Heinrich M, Ankli A, Frei B, Wieman C, Sticher O. 1998. Medicinal plants in Mexico: Healers' consensus and cultural importance. Social Science and Medicine 47:1859-1871.

Heinrich M, Gibbons S. 2001. Ethnopharmacology in drug discovery: an analysis of its role and potential contribution. Journal of Pharmacology 53:425-432.

Hoffman B, Gallaher T. 2007. Importance indices in ethnobotany. Ethnobotany Research and Applications 5:201-218.

Hooker JD. 1872. The Flora of British India Vol. 1. The Flora of British India, Vol. 1

Hong L, Guo A, Huang K, Wei S, Liu B, Meng S, Long C. 2015. Ethnobotanical study on medicinal plants used by Maonan people in China. Journal of Ethnobiology and Ethnomedicine 11:32.
Hussain Al, Anwar F, Sherazi STH, Przybylski R. 2008. Chemical composition, antioxidant and antimicrobial activities of basil (Ocimum basilicum) essential oils depends on seasonal variations. Food chemistry 108(3): 986-995.

Ivanova A, Kostova I, Navas HR, Villegas J. 2004. Volatile components of some Rutaceae species. Zeitschrift für Naturforschung C. 59(3-4): 169-173.

KC M, Phoboo S, Jha PK. 2010. Ecological study of Paris polyphylla Sm. Ecoprint: An International Journal of Ecology 17: 87-93.

Kidane L, Gebremdhin G, Beyene T. 2018. Ethnobotanical study of medicinal plants in Ganda Afeshum District, Eastern Zone of Tigray, Northern Ethiopia. Journal of Ethnobiology and Ethnomedicine 14:64.

Kunwar RM, Nepal BK, Kshhetri HB, Rai SK, Bussmann RW. 2006. Ethnomedicine in Himalaya: a case study from Dolpa, Humla, Jumla and Mustang districts of Nepal. Journal of Ethnobiology and Ethnomedicine 2(1): 27.

Kunwar RM, Shrestha KP, Bussmann RW. 2010. Traditional herbal medicine in Far-West Nepal: a pharmacological appraisal. Journal of Ethnobiology and Ethnomedicine 6(1):35.

Kunwar RM, Mahat L, Acharya RP, Bussmann RW. 2013. Medicinal plants, traditional medicine, markets and management in far-west Nepal. Journal of Ethnobiology and Ethnomedicine 9(1):24.

Kunwar RM, Pandey ML, Kunwar LM, Bhandari A. 2014. Medicinal Plants and Ethnomedicine in Peril: A Case Study from Nepal Himalaya. EvidenceBased Complementary and Alternative Medicine.

Lama YC, SK Ghimire, Aumeeruddy-Thomas Y. 2001. Medicinal plants of Dolpo. Amchis' knowledge and conservation. WWF Nepal Program, Kathmandu, Nepal.

Long C, Li R. 2004. Ethnobotanical studies on medicinal plants used by the Red-Headed Yao People in Jinping, Yunan Province, China. Journal of Ethnopharmacology 90(2-3):389-395.

Luitel DR, Rokaya MB, Timisina B, Munzbergova Z. 2014. Medicinal plants used by the Tamang community in the Makawanpur district of central Nepal. Journal of Ethnobiology and Ethnomedicine 10(1):5.

Mahato RB, Chaudhary RP. 2005. Ethnomedicinal plants of Palpa district, Nepal. Ethnobotany 17(1/2): 152-163.

Malik J, Tauchen J, Landa P, Kutil Z, Marsik P, Kloucek, Kokoska L. 2017. In vitro antiinflammatory 
and antioxidant potential of root extracts from Ranunculaceae species. South African Journal of Botany 109: 128-137.

Malla B, Chhetri RB. 2009. Indigenous knowledge on ethnobotanical plants of Kavrepalanchowk district. Kathmandu University Journal of Science Engineering and Technology 5(2):96-109.

Mahato RB, Chaudhary RP. 2003. Ethnomedicinal Study and Antibacterial activities of selected plants of Palpa district. Nepal. Scientific World 2:38-45

Manandhar NP. 1985. Ethnobotanical notes on certain medicinal plants used by Tharus of Dang Deukhuri district, Nepal. International Journal of Crude Drug Research 23(4):153-259.

Manandhar NP. 1986. Ethnobotany of Jumla district, Nepal. International Journal of Crude Drug Research 24(2):81-89.

Manandhar NP. 1990. Medicinal plant-lore of Tamang tribes of Kabrepalanchowk district, Nepal. Economic Botany 45(1):58-71.

Manandhar NP. 1993: Ethnobotanical note on folklore medicines of Baglung district, Nepal. Contribution to Nepalese Studies 20(2):183-196.

Manandhar NP. 1995a. A survey of medicinal plants of Jajarkot district, Nepal. Journal of Ethnopharmacology 48:1-6.

Manandhar NP. 1995b. Ethnobotanical notes on unexploited wild food plants of Nepal. Ethnobotany 7(1-7):95-101.

Manandhar NP. 2002. Plants and people of Nepal. Timber Press, Portland, Oregon, USA.

Marotti M, Piccaglia R, Giovanelli E, Deans SG, Eaglesham E. 1994. Effects of variety and ontogenic stage on the essential oil composition and biological activity of fennel (Foeniculum vulgare Mill.). Journal of Essential Oil Research 6(1): 57-62.

Napagoda MT, Sundaraperuma T, Fonseka D, Amrasiri S, Gunaratna P. 2018. An ethnobotanical study of the medicinal plants used as antiinflammatory remedies in Gampaha district, Western Province, Sri Lanka. Scientifica.

Obha, H., lokawa, Y, Sharma L. 2008. Flora of Mustang, Nepal. Kodansha Scientific Ltd., Tokyo, Japan

Olsen CS. 2003. Quantification of the trade in medicinal and aromatic plants in and from Nepal. In III WOCMAP Congress on Medicinal and Aromatic Plants-Volume 4: Targeted Screening of Medicinal and Aromatic Plants, Economics 678: 29-35.
Pearce NR, Cribb PJ. 2002. Flora of Bhutan. The Orchids of Bhutan. The Royal Botanic Garden.

Philips O, Gentry AH. 1993. The useful plants of Tambopata, Peru: I. Statistical hypotheses tests with a new quantitative technique. Economic Botany 47(1):15-32.

Polunin O, Stainton A. 1984. Flowers of the Himalaya. Oxford University Press.

Polunin O, Stainton A, Farrer A. 1987. Concise Flowers of the Himalaya. Oxford University Press.

Press JR, Shrestha KK, Sutton DA. 2000. Annotated Checklist of the Flowering Plants of Nepal. The Natural History Museum, London.

Rajbhandari KR. 2001. Ethnobotany of Nepal. Ethnobotanical Society of Nepal. Kishor Offset Press, Kathmandu.

Rajbhandari KR, Rai SK. 2017. A Handbook of the Flowering Plants of Nepal Volume 1. Government of Nepal, Ministry of Forests and Soil Conservation, Department of Plant Resources, Kathmandu, Nepal.

Rokaya MB, Münzbergová Z, Timsina B. 2010. Ethnobotanical study of medicinal plants from the Humla district of western Nepal. Journal of Ethnopharmacology 130: 485-504.

Rokaya MB, Uprety Y, Poudel RC, Timsina B, Münzbergová Z, Asselin H, Sigdel SR. 2014. Traditional uses of medicinal plants in gastrointestinal disorders in Nepal. Journal of Ethnopharmacology 158: 221-229.

Rossato SC, Leitão-Filho HF, Begossi A. 1999. Ethnobotany of Caiçaras of the Atlantic forest coast (Brazil). Economic Botany 53:387-395.

Sapkota PP. 2008. Ethno-ecological Observation of Magar of Bukini, Baglung, Western, Nepal. Dhaulagiri Journal of Sociology and Anthropology 2: 227-252.

Saslis-Lagoudakis $\mathrm{CH}$, Hawkins JA, Greenhill SJ, Pendry CA, Watson MF, Tuladhar-Douglas W, Baral SR, Savolainen V. 2014. The evolution of traditional knowledge: environment shapes medicinal plant use in Nepal. Proceedings of the Royal Society B: Biological Sciences 281(1780):20132768.

Sathiyaraj G, Muthukumar T, Ravindran KC. 2015. Ethnomedicinal importance of fern and fern allies traditionally used by tribal people of Palani Hills (Kodaikanal), Western Ghats, South India. Journal of Medicinal Herbs and Ethnomedicine 1:4-9. doi: 10.5455/jmhe.2015-07-08.

Schults RE. 1962. The role of ethnobotanist in the search of new medicinal plants. Lloydia 25(4): 45-64. 
Shrestha I, Pradhan N. 1986. Medicinal Plants of Chobhar Village of Kathmandu district, Nepal. Journal of Natural History Museum (Kathmandu) 10(1-4):65-72.

Shrestha KK, Ghimire SK, Gurung TN, Lama YC, Aumeeruddy Y. 1998. Conservation of Plant Resources, Community Development and Training in Applied Ethnobotany at Shey-Phoksundo National Park and its Buffer zone, Dolpa. WWF/Nepal Program Report Series. (33).

Shrestha KK, Bhattarai S, Bhandari P. 2018. Handbook of flowering plants of Nepal: Scientific Publishers, Jodhpur, India.

Shrestha PM, Dillion SS. 2003. Medicinal plant diversity and use in the highlands of Dolakha district, Nepal. Journal of Ethnopharmacology. 86(1): 81-96.

Singh AG, Kumar A, Tewari DD. 2012. An ethnobotanical survey of medicinal plants used in Terai forest of western Nepal. Journal of Ethnobiology and Ethnomedicine 8(1):19.

Singh AG, Kumar A, Tewari, DD Bharati, KA. 2018. New ethnomedicinal claims from Magar community of Palpa district, Nepal. Indian Journal of Traditional Knowledge 17(3): 499-511. http://nopr.niscair.res.in/handle/123456789/44583

Srithi K, Balslevb $\mathrm{H}$, Wangpakapattanawonga $\mathrm{P}$, Srisangac P, Trisonthia C. 2009. Medicinal plant knowledge and its erosion among the Mien (Yao) in northern Thailand. Journal of Ethnopharmacology 123:335-342.

Stainton A, Polunin O. 1988. Flowers of the Himalaya. Oxford University Press.

Strohl WR. 2000. The role of natural products in a modern drug discovery program. Drug discovery today 5(2): 39-41.

Tardio J, Santayana MPD. 2008. Cultural importance indices: A comparative analysis based on the useful wild plants of Southern Cantabrai (Northern Spain). Economic Botany 62(1): 24-39.

Thapa S. 2012. Medico-ethnobotany of Magar community in Salija VDC of Parbat district, central Nepal. Our Nature 10(1): 176-190.

Trotter R, Logan M. 1986. Informant consensus: a new approach for identifying potentially effective medicinal plants. In: EtkinGh NL. Editor. Plants in indigenous medicine and diet: biobehavioral approaches. Bedford Hills, NY: Redgraves publishers. 91-112.

Uprety Y, Asselin H, Boon EK, Yadav S, Shrestha KK. 2010. Indigenous use and bio-efficacy of medicinal plants in the Rasuwa District, Central
Nepal. Journal of Ethnobiology and Ethnomedicine 6(3).

Uprety Y, Paudel RC, Shrestha KK, Rajbhandary S, Tiwari NN, Shrestha UB, Asselin H. 2012. Diversity of use and local knowledge of wild edible plant resources in Nepal. Journal of Ethnobiology and Ethnomedicine 8:16.

UNEP [United Nations Environment Program]. 2012. Green Economy Sectorial Study: BiotradeHarnessing the Potential for Transitioning to a Green Economy-The Case of Medicinal and Aromatic Plants in Nepal. United Nations Environment Program; Nairobi, Kenya.

WHO. 2011. The World Traditional Medicines Situation, in Traditional medicines: Global Situation, Issues and Challenges. Geneva 3:1-14. 


\section{Additional file 1. Ethnobotanical Data Collection in Thabang VDC, Rolpa District}

Demographic information

1. Name of (a) informant(s).
(b). Age
(c) Sex: Male
Female
(d) VDC............................ Ward no.:........ Tole:........

(e) How long have you been living in this area?

i) Since 10 years ii) Since 30 years iii) Since 50 yearsiv) More than 50 years

f) Main occupation

Category :-

Agriculture $=1 ;$ Services $=2 ;$ Business $=3 ;$ Student $=4 ;$ Wagelabour $=5 ;$ Other (Specify).

2. Ethnobotanical knowledge of respondent on different plant groups

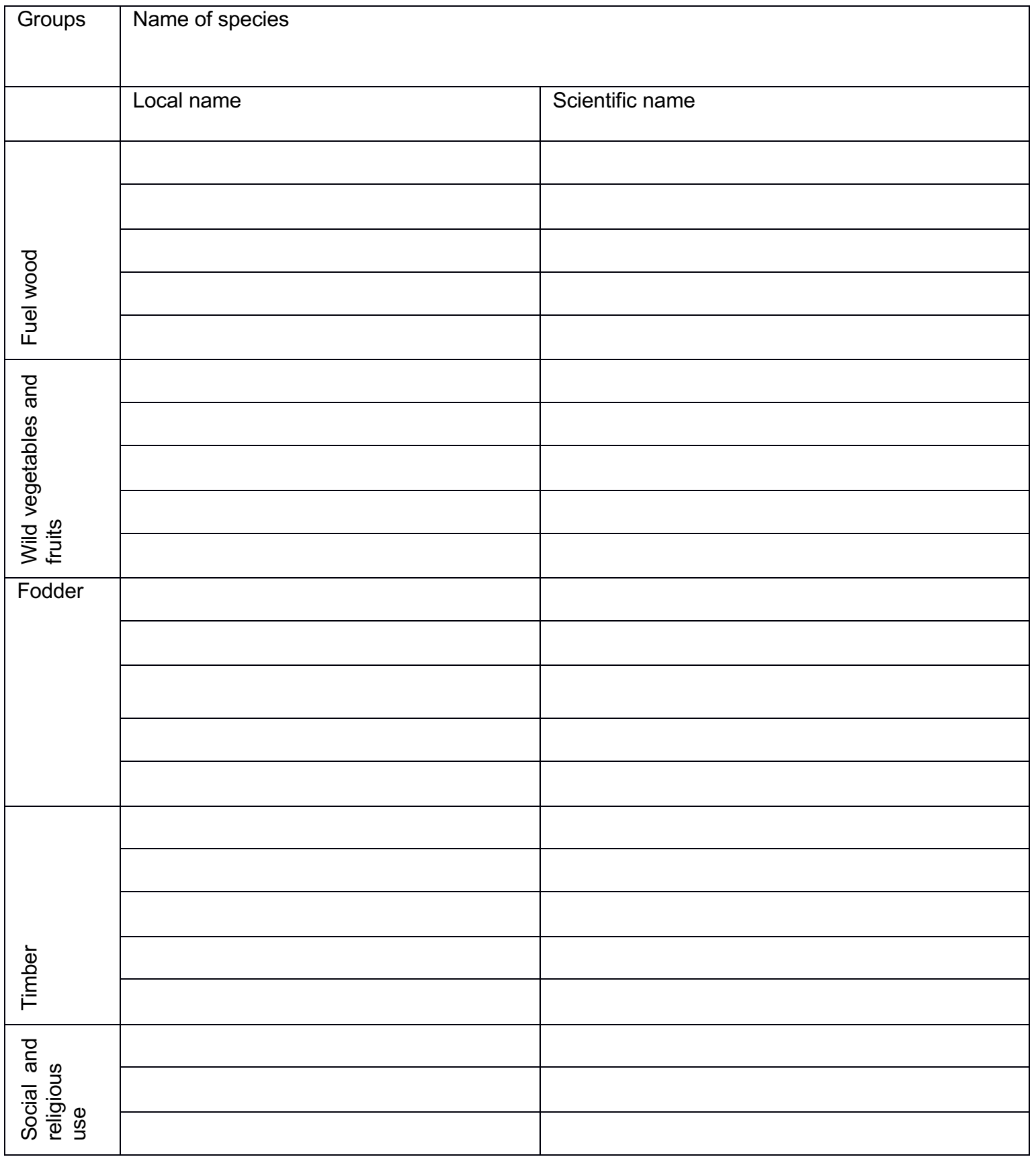




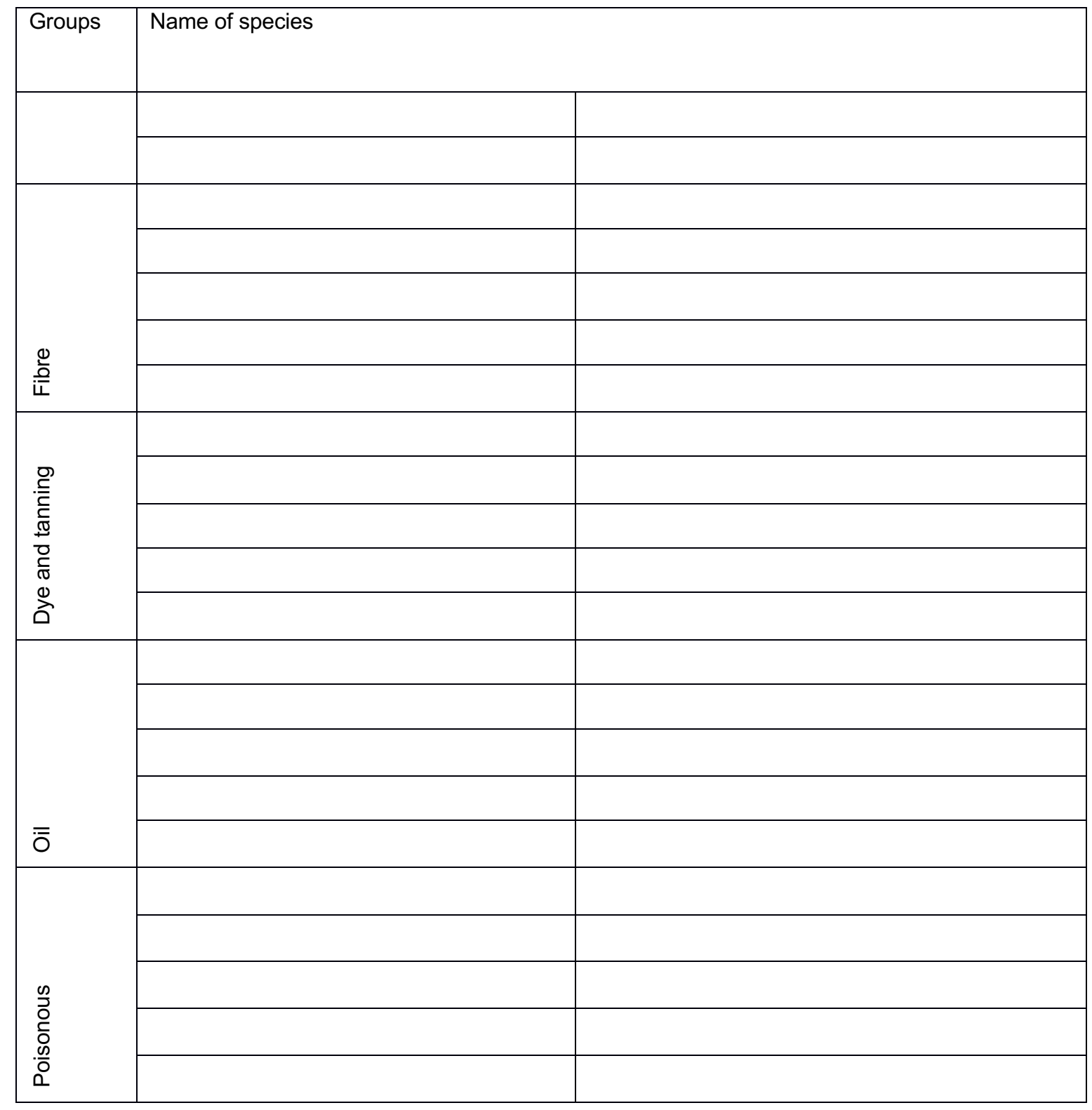

3. Ethnobotanical knowledge of respondent on Medicinal plants.

\begin{tabular}{|c|c|c|c|c|c|c|c|c|c|c|}
\hline \multicolumn{2}{|c|}{ Name of of the plant } & \multirow{2}{*}{$\begin{array}{ll}\text { Name of } \\
\text { disorder }\end{array}$} & \multirow[t]{2}{*}{ Symptoms } & \multicolumn{6}{|c|}{ Plant parts used } & \multirow{2}{*}{$\begin{array}{l}\text { Mode of } \\
\text { use }\end{array}$} \\
\hline Local name & Latin name & & & 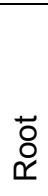 & $\frac{+}{\circ}$ & 离 & 产 & 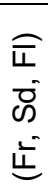 & $\begin{array}{l}\frac{0}{0} \\
\frac{1}{5} \\
\frac{\pi}{2}\end{array}$ & \\
\hline & & & & & & & & & & \\
\hline & & & & & & & & & & \\
\hline & & & & & & & & & & \\
\hline & & & & & & & & & & \\
\hline & & & & & & & & & & \\
\hline
\end{tabular}

4. Why do you choose plants from that locality?

Ans.

5. Mostly which staged plants are preferred for harvest?

Ans

6. How can these plants be preserved?

Ans. 